\title{
Multiband OFDM for Covert Acoustic Communications
}

\author{
Geert Leus and Paul A. van Walree
}

\begin{abstract}
A multiband OFDM transmitter and receiver are presented for underwater communications at low SNR. Compared with a single-band OFDM scheme, the multiband approach leads to a considerable reduction in the receiver complexity. The proposed system has been tested at sea with 16 subbands covering a total bandwidth of $3.6 \mathrm{kHz}$, at user data rates of 4.2 and $78 \mathrm{bit} / \mathrm{s}$, and over ranges up to $52 \mathrm{~km}$. At the lower rate, successful message recovery is achieved on a single hydrophone at SNRs down to $-17 \mathbf{d B}$ in a benign channel. In channels with a severe delay-Doppler spread the critical SNR rises by some $4 \mathrm{~dB}$. At $78 \mathrm{bit} / \mathrm{s}$ the limits of the OFDM signaling scheme are clearly revealed, but at $4.2 \mathrm{bit} / \mathrm{s}$ the performance is limited by failure of signal detection and initial synchronization.
\end{abstract}

Index Terms-Underwater communications, covert communications, multiband OFDM, time-varying systems.

\section{INTRODUCTION}

$\mathbf{T}$ HE JOINT European research project UUV Covert Acoustic Communications (UCAC) aims at the establishment of a covert communication link between unmanned underwater vehicles (UUVs) and a distant mother platform. Literature on covert underwater communications is scarce, with the characteristics of the acoustic propagation channel being cited as a major obstacle [1], [2]. There is no unique definition of covertness, and the word may be used to indicate, for example, encryption [3], spatial scrambling of the sound [4], or the use of marine mammal sounds [5]. For the present study the task is simply to communicate at a low signal-tonoise ratio (SNR), thereby hiding messages in the ambient noise and reducing the probability of detection by third parties. Auditive aspects are also important, with noiselike waveforms such as phase-shift keying (PSK) and orthogonal frequency-division multiplexing (OFDM) being preferred to frequency-shift keyed or chirp modulations. Direct-sequence spread spectrum is a logical candidate modulation to achieve acoustic data transfer at low SNR [6], [7], but the present paper shows that OFDM is a strong contender.

OFDM is generally considered in wireless communications systems to simplify the equalization process. The coherence time of radio propagation channels is usually much longer than the symbol period, allowing for a simple one-tap frequencydomain channel equalizer. In contrast, underwater acoustic communication is often subject to considerable Doppler

Manuscript received February 21, 2008; revised August 11, 2008. This work was performed under the EUROPA MOU ERG No1, RTP 110.060 "UUV Covert Acoustic Communications". The Dutch contribution was sponsored by the Defence Research and Development Department of the Netherlands Ministry of Defence. Geert Leus is supported in part by NWO-STW under the VIDI program (DTC.6577).

Geert Leus is with Delft University of Technology - Fac. EEMCS, Mekelweg 4, 2628 CD Delft, The Netherlands (e-mail: g.j.t.leus@tudelft.nl).

Paul van Walree is with Netherlands Organisation for Applied Scientific Research TNO, Oude Waalsdorperweg 63, 2509 JG The Hague, The Netherlands. (e-mail: paul.vanwalree@tno.nl).

Digital Object Identifier 10.1109/JSAC.2008.081206. spreading, and the channel coherence time may compare with the symbol period. This causes a loss of orthogonality among the carriers and leads to intercarrier interference (ICI). But even then, OFDM equalization can still be implemented at relatively low complexity by means of sophisticated algorithms that exploit the banded structure of the frequency-domain channel matrix [8]-[11]. The use of OFDM in underwater communications is not new, see for example [12]-[16]. In [13], a low-complexity OFDM scheme is presented, where ICI is ignored and the phase of every carrier is adaptively tracked with a single Doppler rate parameter. However, for large Doppler spreads and/or longer OFDM symbols, this method loses track of the channel. In [14]-[16], on the other hand, a more advanced OFDM scheme is proposed, where ICI is not ignored and the channel is modeled as a time-varying channel with a single Doppler rate parameter. This model has the interesting characteristic that Doppler compensation reduces the time variability of the channel to a single carrierfrequency offset (CFO) [15], which allows the use of simple channel estimation and equalization methods [14], and leads to interesting scaling features [16]. However, although ICI is not ignored, this channel model is no longer valid for large Doppler spreads and/or long OFDM symbols. Compared with the above existing works, the present paper contains a number of novel contributions:

- The long delay spread encountered in littoral areas requires a long OFDM symbol duration in order to limit the redundancy of the cyclic prefix, which should have a length on the order of the maximal (expected) delay spread. However, this may result in a considerable channel time variability within a single OFDM symbol, leading to ICI. We deal with this problem by modeling and estimating the channel within a single OFDM symbol as a basis expansion model (BEM) [17], [18] after Doppler compensation. Note that a BEM is more general than the CFO used in [14]-[16], and it allows us to model more complicated dynamic scenarios. This comes at the price of an increased channel estimation and equalization complexity.

- Modeling the channel variability as a CFO after Doppler compensation leads to simple channel estimation and equalization procedures [14]. When a BEM channel is considered, however, the related procedures get more complex. But fortunately, in that case, the receiver complexity can be considerably reduced by a multiband OFDM approach, where the available frequency band is divided into smaller subbands, each of which is modulated by OFDM.

- We aim at covert communications and thus we rely on coding and spreading operations to allow for reliable 
communications at a low SNR. Moreover, at the receiver, we do not view channel equalization and despreading as two separate linear operations, but we combine them using a joint approach.

Note that multiband OFDM has also been considered for ultra wideband (UWB) communications [19]. In that case, every transmission only occupies one subband at the time and it hops from one subband to another. Hence, complexity savings are then obtained through reduction of the instantaneous bandwidth. In the proposed multiband OFDM scheme, all subbands are constantly occupied. The reduction in complexity then comes from the fact that the receiver complexity for highly time-varying environments decreases with the number of subbands, as shown in Section IV.

Notation: We use upper (lower) case bold face letters to denote matrices (column vectors). $(\cdot)^{*},(\cdot)^{T},(\cdot)^{H}$, and $(\cdot)^{\dagger}$, represent conjugate, transpose, complex conjugate transpose (Hermitian), and pseudo inverse (Moore-Penrose inverse), respectively. $\lfloor x\rfloor$ and $\lceil x\rceil$ round $x$ toward the nearest integer smaller than or equal to $x$ and larger than or equal to $x$, respectively, and $\Re(x)$ denotes the real part of $x . \mathrm{E}(\cdot)$ stands for the expectation, $\otimes$ represents the Kronecker product, * denotes convolution, and $\circledast$ circular convolution. $a \bmod b$ gives the remainder of $a$ divided by $b \cdot \operatorname{diag}(\mathbf{x})$ stands for a diagonal matrix with $\mathbf{x}$ as diagonal, and $\operatorname{diag}\left(\mathbf{X}_{0}, \ldots, \mathbf{X}_{N-1}\right)$ for the block diagonal matrix with $\mathbf{X}_{0}, \ldots, \mathbf{X}_{N-1}$ as diagonal blocks. We use $[\mathbf{x}]_{n}$ to indicate the $n$-th element of $\mathbf{x}$ (counting from 0$)$, and $[\mathbf{X}]_{n, m}$ to indicate the $(n, m)$-th entry of $\mathbf{X}$ (counting from 0 ). Further, $[\mathbf{x}]_{n: n^{\prime}}$ indicates the subvector of $\mathbf{x}$ from element $n$ to $n^{\prime}$ (counting from 0 ) and $[\mathbf{X}]_{n: n^{\prime}, m: m^{\prime}}$ indicates the submatrix of $\mathbf{X}$ from row $n$ to $n^{\prime}$ and from column $m$ to $m^{\prime}$ (counting from 0 ), where only the colon is kept when all rows or columns are included (Matlab notation). Next, $\mathbf{I}_{N}$ denotes the $N \times N$ identity matrix and $\mathbf{0}_{M \times N}$ the $M \times N$ all-zero matrix. Finally, $\mathbf{F}_{N}$ stands for the unitary $N$-point discrete Fourier transform (DFT) matrix with $\left[\mathbf{F}_{N}\right]_{n, m}=\frac{1}{\sqrt{N}} \mathrm{e}^{-\iota \frac{2 \pi}{N} n m}$, where we define $\iota=\sqrt{-1}$.

\section{ACQUisition, SynChronizATion, AND DATA MOdel}

In this section we will briefly discuss acquisition and synchronization, and the data model that results from these operations. This data model will be the starting point of our multiband OFDM approach, which is discussed in the next sections.

Acquisition and synchronization rely on the transmission of a template consisting of a maximal-length sequence modulated on sinc pulses. At the receiver, the hydrophone signal is split up in segments with a length of several seconds. Each successive segment is rms-normalized and correlated with a bank of different Doppler-shifted (resampled) versions of the template. The peak filter output is obtained across all Doppler branches, and as soon as it crosses a certain threshold the signal is considered acquired with corresponding delay and Doppler shifts. Following detection, the template is stripped off and the remaining signal is delay-Doppler shifted such that the strongest multipath arrival is centered in the causal impulse response representing the acoustic channel.

For the subsequent developments, we move to a digital bandpass data model at rate $1 / T$, much larger than the signal bandwidth. This rate $1 / T$ could for instance be the rate required for the transmitter, or the digitization rate at the receiver. Transitions to and from another rate can easily be incorporated by means of a resampling operation. The adopted data model reads

$$
\bar{v}[n]=\sum_{l=0}^{L_{h}-1} \bar{h}[n ; l] \bar{u}[n-l]+\bar{\varepsilon}[n],
$$

where $\bar{u}[n]$ is the transmitted signal, $\bar{v}[n]$ is the received signal, $\bar{\varepsilon}[n]$ represents the additive noise, and $\bar{h}[n ; l]$ models the remaining acoustic channel as a causal time-varying finite impulse response (FIR) filter with length $L_{h}$, which has a centered chief multipath arrival ${ }^{1}$. Notice that although such a model is generally adopted for narrowband systems, it also holds for wideband systems because the time variation of the FIR filter can capture this wideband nature. Other timevarying effects that can be modeled by $\bar{h}[n ; l]$ are transmitter and receiver motion as well as the reflections off moving scatterers or the moving sea surface. The data model (1) is the starting point of our multiband OFDM approach. For ease of presentation, we assume in the following sections that the acquisition and synchronization steps have already been carried out and that the data transmission can start at time instant $n=0$.

\section{TRANSMITTER}

In this section we introduce the transmitter operations of the proposed multiband OFDM approach. Fig. 1 depicts the overall transmitter structure, while Fig. 2 details the subband processing block of Fig. 1. A bit sequence is first turbo encoded and modulated onto a PSK alphabet. The resulting data symbols are then parsed into blocks of length $K_{\mathrm{d}}$. Each block of $K_{\mathrm{d}}$ data symbols is interleaved with a block of $K_{\mathrm{t}}$ training symbols (pilots), leading to a block of $K=K_{\mathrm{d}}+K_{\mathrm{t}}$ symbols, where $K$ is the number of carriers used in an OFDM symbol. We will have a total of $P$ such blocks of $K$ symbols, denoted by a sequence of $K \times 1$ vectors $\mathbf{s}_{p}, p=0,1, \ldots, P-1$. We assume that every vector $\mathbf{s}_{p}$ has energy $K$. Specific pilot design for channel estimation will be discussed in more detail in the next section. These $P$ vectors $\mathbf{s}_{p}$ will now be spread out over $J$ subbands with $I$ OFDM symbols each. The spreading is done in such a way that all slots are occupied by a single vector, which means that the spreading factor is given by $I J / P$. The spreading is simply realized by a repetition code, although any other spreading code could be used. Denoting the $i$-th OFDM symbol of the $j$-th subband by the $K \times 1$ vector $\mathbf{s}_{i}^{(j)}$, with $i=0,1, \ldots, I-1$ and $j=0,1, \ldots, J-1$, we will assume that $\mathbf{s}_{p}$ is repeated over the set $\left\{\mathbf{s}_{i}^{(j)}\right\}$ with indices $(i, j) \in \mathcal{I}_{p}$, i.e., $\mathbf{s}_{i}^{(j)}=\mathbf{s}_{p}$ if $(i, j) \in \mathcal{I}_{p}$. And since all slots are occupied by a single vector, we have $\bigcup_{p=0}^{P-1} \mathcal{I}_{p}=\{(i, j) \mid i=0,1, \ldots, I-1, j=0,1, \ldots, J-1\}$ and $\mathcal{I}_{p} \cap \mathcal{I}_{p^{\prime}}=\emptyset$, if $p \neq p^{\prime}$.

Every $K \times 1$ OFDM symbol $\mathbf{s}_{i}^{(j)}$ is transformed into an $N \times 1$ baseband time domain vector $\mathbf{x}_{i}^{(j)}$ with samples at a rate of $1 / T$. This can be done by upsampling followed by an ideal lowpass filtering, which is implicitly carried out by

\footnotetext{
${ }^{1}$ We use a bar to indicate that the signals are passband signals.
} 


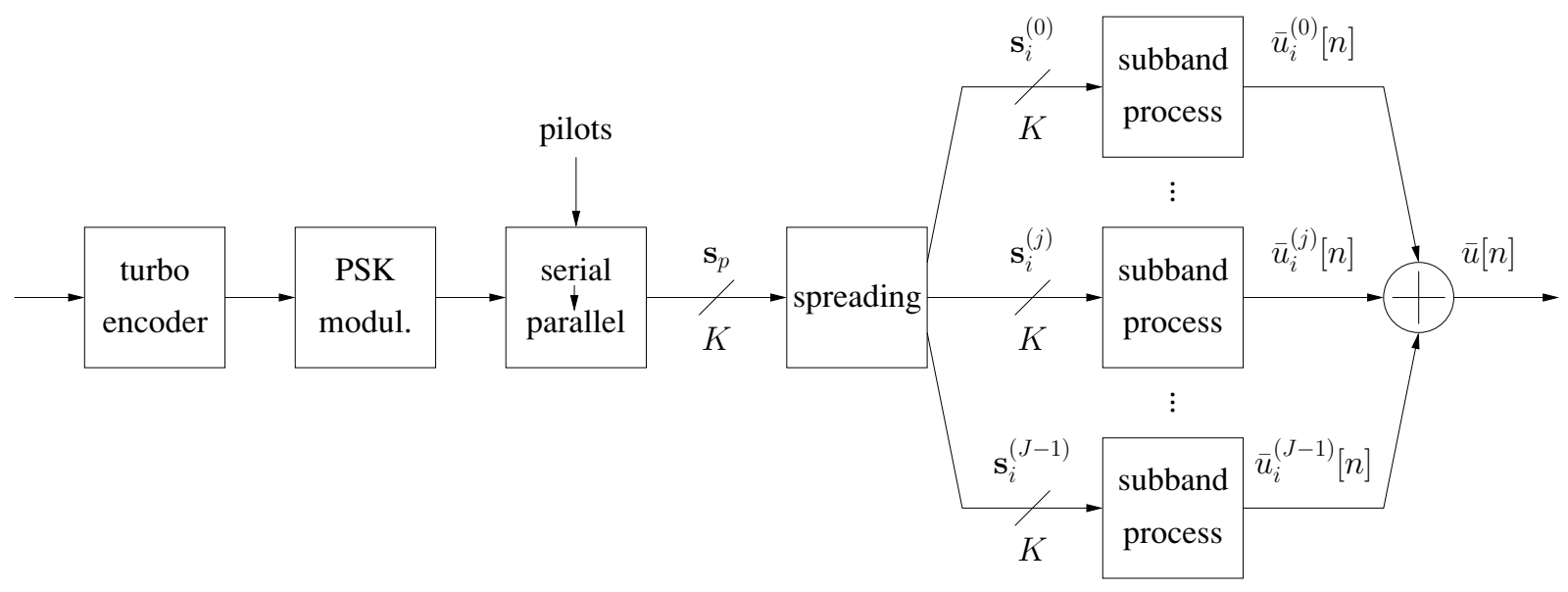

Fig. 1. Block diagram of the transmitter operations.

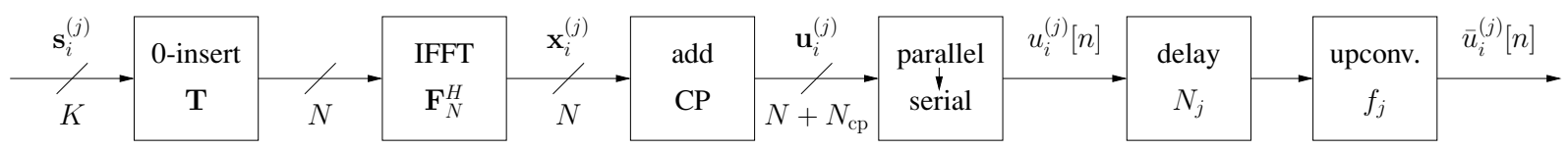

Fig. 2. Block diagram of the subband processing at the transmitter.

inserting $N-K$ zeros in the middle of $\mathbf{s}_{i}^{(j)}$ and transforming the result by means of an $N$-point unitary inverse DFT (IDFT):

$$
\mathbf{x}_{i}^{(j)}=\mathbf{F}_{N}^{H} \mathbf{T} \mathbf{s}_{i}^{(j)}
$$

where $\mathbf{T}$ is the $N \times K$ zero-inserting matrix $\mathbf{T}=$ $\left[\left[\mathbf{I}_{K / 2}, \mathbf{0}_{K / 2 \times K / 2}\right]^{T}, \mathbf{0}_{K, N-K},\left[\mathbf{0}_{K / 2 \times K / 2}, \mathbf{I}_{K / 2}\right]^{T}\right]^{T}$. Indeed, as explained in Appendix A, the operation (2) can be interpreted as transforming $\mathbf{s}_{i}^{(j)}$ by means of a $K$-point unitary IDFT, then upsampling it with a factor $N / K$, and finally circularly filtering it with an ideal lowpass filter $c[n]$ with frequency response $\tilde{c}[n]$. See Appendix A for the exact expressions of $c[n]$ and $\tilde{c}[n]$. Performing these steps explicitly would significantly increase the computational complexity. It could be reduced by shortening the filter length, but then one looses ideality and one has to provide additional guards in the frequency domain to take care of the leakage from one subband into another. Hence, in view of all these issues, we have chosen for the implicit approach.

In the next step, a cyclic prefix (CP) of $N_{\mathrm{cp}}$ samples is added, which results in the $\left(N+N_{\mathrm{cp}}\right) \times 1$ vector $\mathbf{u}_{i}^{(j)}=$ $\left[\left[\mathbf{x}_{i}^{(j)}\right]_{N-N_{\mathrm{cp}}: N-1}^{T}, \mathbf{x}_{i}^{(j) T}\right]^{T}$. The reason for this CP is that it simplifies the equalization process, since if the channel (including all delay effects) fits within the $\mathrm{CP}$, the channel in (1) resembles a quasi-circular convolution at the receiver ${ }^{2}$, and a single input vector will be related to only one output vector.

OFDM waveforms normally have a high peak-to-average power ratio (PAPR). The use of identical pilots in every vector, and the simultaneous occurrence of two or more identical vectors $\mathbf{s}_{p}$ in two or more subbands, further increases the PAPR. This limits the power output of the employed transducers, which is not a major drawback in the context of covertness, but which limits the maximum range. To

\footnotetext{
${ }^{2}$ We use the term "quasi-circular" for the generalization of the term "circular" to time-varying filters.
}

contain the PAPR of the compound waveform, the last two operations on $\mathbf{u}_{i}^{(j)}$, which we rewrite for convenience as $\mathbf{u}_{i}^{(j)}=\left[u_{i}^{(j)}[0], \ldots, u_{i}^{(j)}\left[N+N_{\mathrm{cp}}-1\right]\right]^{T}$, insert a timing offset of $N_{j}$ samples, and modulate the signal onto a carrier of frequency $f_{j}$, chosen such that the subbands will not overlap. These operations lead to the following contribution of the $i$-th OFDM symbol of the $j$-th subband to the transmitted signal $\bar{u}[n]$ :

$$
\bar{u}_{i}^{(j)}[n]=\left\{\begin{array}{c}
\sqrt{\frac{2 E}{K N}} \Re\left[u_{i}^{(j)}\left(n-n_{i}^{(j)}\right) \exp \left(\iota 2 \pi f_{j} n T\right)\right], \\
\quad \text { if } n=n_{i}^{(j)}, \ldots, n_{i}^{(j)}+N+N_{\mathrm{cp}}-1 \\
0, \quad \text { otherwise }
\end{array},\right.
$$

where $n_{i}^{(j)}=N_{j}+i\left(N+N_{\mathrm{cp}}\right)$ and $E / T$ is the average power per subband. The overall transmitted sequence can then be written as

$$
\bar{u}[n]=\sum_{i=0}^{I-1} \sum_{j=0}^{J-1} \bar{u}_{i}^{(j)}[n],
$$

with an average power of $J E / T$. This sequence is finally applied at the input of the transmitter.

\section{RECEIVER}

Let us assume that acquisition and synchronization have been carried out as indicated in Section II, so that we can adopt the data model (1). We then have to carry out in reverse the steps that were taken at the transmitter, followed by channel estimation, equalization and despreading, and turbo decoding. The overall receiver structure is illustrated in Fig. 3, with Fig. 4 detailing the operations carried out in each subband. In order to decode the $i$-th OFDM symbol of the $j$ th subband, we first select the correct $N+N_{\mathrm{cp}}$ samples from $\bar{v}[n]$, taking into account the timing offset of $N_{j}$ samples, and remove the 


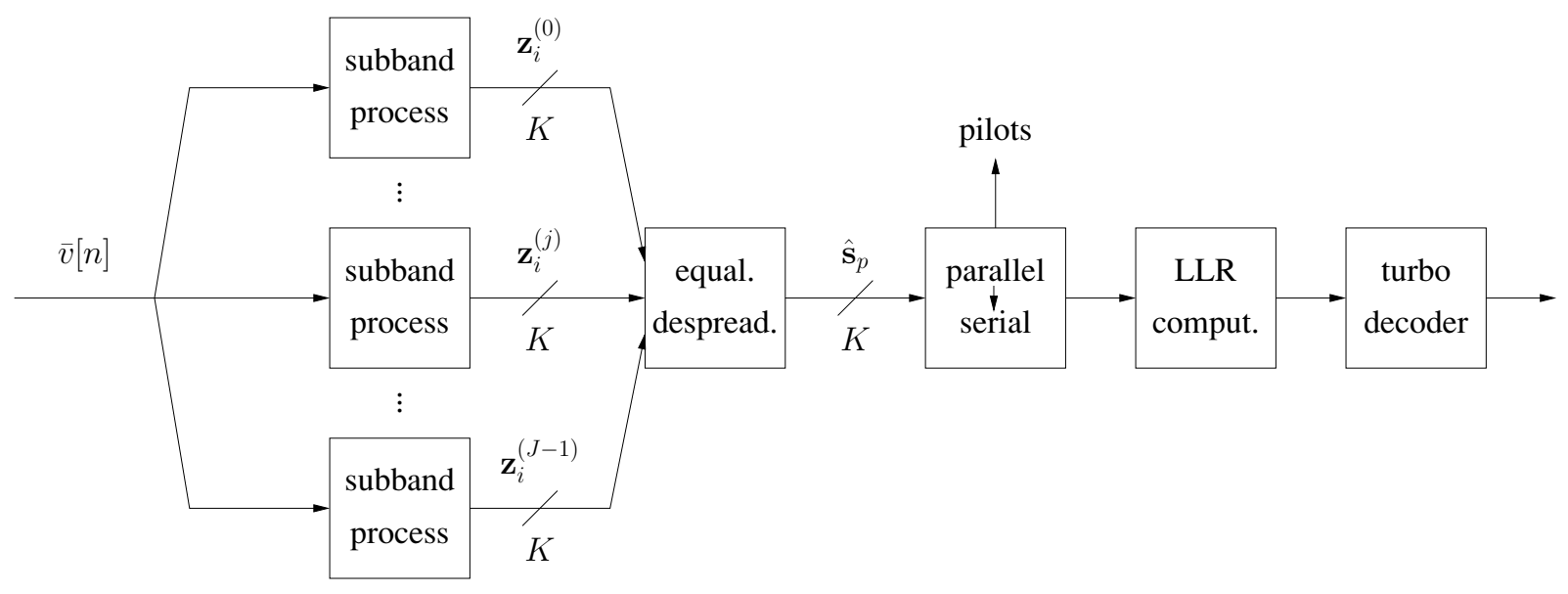

Fig. 3. Block diagram of the receiver operations.

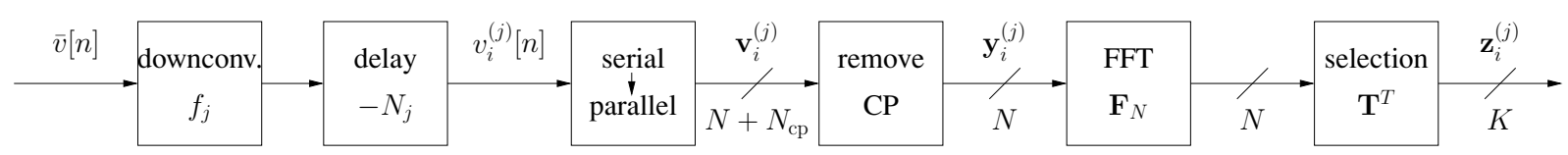

Fig. 4. Block diagram of the subband processing at the receiver.

carrier $f_{j}$. In other words, we construct the following signal:

$$
\begin{aligned}
& v_{i}^{(j)}\left[n-n_{i}^{(j)}\right]= \\
& \left\{\begin{array}{l}
\sqrt{\frac{2 K N}{E}} \bar{v}[n] \exp \left(-\iota 2 \pi f_{j} n T\right), \\
\text { if } n=n_{i}^{(j)}, \ldots, n_{i}^{(j)}+N+N_{\mathrm{cp}}-1 \\
0, \quad \text { otherwise }
\end{array},\right.
\end{aligned}
$$

where we normalize the output power with respect to the input power. We implicitly assume that an ideal lowpass filtering is carried out at this point in order to remove the double frequencies, and reach an equivalent baseband representation. Next the CP is stripped from the $\left(N+N_{\mathrm{cp}}\right) \times 1$ vector $\mathbf{v}_{i}^{(j)}=\left[v_{i}^{(j)}[0], \ldots, v_{i}^{(j)}\left[N+N_{\mathrm{cp}}-1\right]\right]^{T}$, which is reduced to the $N \times 1$ vector $\mathbf{y}_{i}^{(j)}=\left[\mathbf{v}_{i}^{(j)}\right]_{N_{\mathrm{cp}}: N+N_{\mathrm{cp}}-1}$.

In Appendix $\mathrm{B}$, we show that if i) the CP length $N_{\mathrm{cp}}$ is large enough for the channel (including all delay effects) to fit within the CP for every subband, i.e., $N_{\mathrm{cp}} \geq L_{h}-1+$ $\max _{j, j^{\prime}}\left(N_{j}-N_{j^{\prime}}\right)$, ii) the different carriers $f_{j}$ are separated by $(K+G) /(N T)$, where $1 /(N T)$ is the carrier spacing and $G /(N T)$ is some frequency guard between the subbands, and iii) the guard $G /(N T)$ is larger than or equal to the maximal Doppler spread $f_{\mathrm{D}}$, then $\mathbf{y}_{i}^{(j)}$ only depends on the $i$-th OFDM symbol of the $j$-th subband if the $K$ carriers of interest are chosen. Hence, the multiband interference can be completely removed by ideal lowpass filtering followed by downsampling, which is implicitly carried out by transforming the $N \times 1$ baseband time-domain vector $\mathbf{y}_{i}^{(j)}$ by means of an $N$-point unitary DFT, and selecting the first $K / 2$ and last $K / 2$ carriers, which yields the $K \times 1$ vector $\mathbf{z}_{i}^{(j)}$

$$
\begin{aligned}
\mathbf{z}_{i}^{(j)} & =\mathbf{T}^{T} \mathbf{F}_{N} \mathbf{y}_{i}^{(j)} \\
& =\mathbf{T}^{T} \mathbf{F}_{N}\left(\mathbf{H}_{i}^{(j)} \mathbf{x}_{i}^{(j)}+\boldsymbol{\zeta}_{i}^{(j)}\right)
\end{aligned}
$$

where $\boldsymbol{\zeta}_{i}^{(j)}$ is similarly defined from $\bar{\varepsilon}[n]$ as $\mathbf{y}_{i}^{(j)}$ from $\bar{v}[n]$, and where $\mathbf{H}_{i}^{(j)}$ is the $N \times N$ quasi-circular channel matrix of interest given by $\left[\mathbf{H}_{i}^{(j)}\right]_{n, n^{\prime}}=h_{i}^{(j)}\left[n ;\left(n-n^{\prime}\right) \bmod N\right]$ with $h_{i}^{(j)}[n ; l]=\bar{h}\left[n_{i}^{(j)}+N_{\mathrm{cp}}+n ; l\right] \exp \left(-\iota 2 \pi f_{j} l T\right)$. Indeed, similar to Appendix A, it can be shown that (6) can be interpreted as first circularly filtering $\mathbf{y}_{i}^{(j)}$ with the ideal lowpass filter $c[n]$ with frequency response $\tilde{c}[n]$ (see previous section), then downsampling it by a factor $N / K$, and finally transforming it by means of a $K$-point unitary DFT. For the same reasons as before, we do not perform these steps in an explicit way.

In Appendix $\mathrm{C}$, it is finally shown that the alternative interpretations of (2) and (6) allow us to relate $\mathbf{z}_{i}^{(j)}$ to $\mathbf{s}_{i}^{(j)}$ as

$$
\begin{aligned}
\mathbf{z}_{i}^{(j)} & =\mathbf{T}^{T} \mathbf{F}_{N} \mathbf{H}_{i}^{(j)} \mathbf{F}_{N}^{H} \mathbf{T} \mathbf{s}_{i}^{(j)}+\boldsymbol{\eta}_{i}^{(j)} \\
& =\mathbf{F}_{K} \boldsymbol{\Lambda}_{i}^{(j)} \mathbf{F}_{K}^{H} \mathbf{s}_{i}^{(j)}+\boldsymbol{\eta}_{i}^{(j)}
\end{aligned}
$$

where $\boldsymbol{\eta}_{i}^{(j)}$ is similarly defined from $\boldsymbol{\zeta}_{i}^{(j)}$ as $\mathbf{z}_{i}^{(j)}$ from $\mathbf{y}_{i}^{(j)}$, and $\boldsymbol{\Lambda}_{i}^{(j)}$ is the $K \times K$ quasi-circular channel matrix given by $\left[\boldsymbol{\Lambda}_{i}^{(j)}\right]_{k, k^{\prime}}=\lambda_{i}^{(j)}\left[k ;\left(k-k^{\prime}\right) \bmod K\right]$ with $\lambda_{i}^{(j)}[k ; l]$ the $N / K$-times downsampled version of $c[n] \circledast h_{i}^{(j)}[n ; l] \circledast c[n]$. Using frequency-domain matrices, we obtain

$$
\begin{aligned}
\mathbf{z}_{i}^{(j)} & =\mathbf{T}^{T} \tilde{\mathbf{H}}_{i}^{(j)} \mathbf{T s}_{i}^{(j)}+\boldsymbol{\eta}_{i}^{(j)} \\
& =\tilde{\Lambda}_{i}^{(j)} \mathbf{s}_{i}^{(j)}+\boldsymbol{\eta}_{i}^{(j)}
\end{aligned}
$$

where $\tilde{\mathbf{H}}_{i}^{(j)}=\mathbf{F}_{N} \mathbf{H}_{i}^{(j)} \mathbf{F}_{N}^{H}$ and $\tilde{\Lambda}_{i}^{(j)}=\mathbf{F}_{K} \boldsymbol{\Lambda}_{i}^{(j)} \mathbf{F}_{K}^{H}$. Note that the Doppler spread limitation of $h_{i}^{(j)}[n ; l]$, which leads to a circularly banded frequency-domain channel matrix $\tilde{\mathbf{H}}_{i}^{(j)}$ (see Appendix B), carries over to the Doppler spread limitation of $\lambda_{i}^{(j)}[k ; l]$ and leads to a circularly banded frequency-domain channel matrix $\tilde{\Lambda}_{i}^{(j)}$. This is also clear from (8), where we can see that $\tilde{\Lambda}_{i}^{(j)}$ simply is a submatrix of $\tilde{\mathbf{H}}_{i}^{(j)}$. Hence, if $\tilde{\mathbf{H}}_{i}^{(j)}$ has a bandwidth $B$, then $\tilde{\Lambda}_{i}^{(j)}$ has a bandwidth $B$. Notice that this 
bandwidth even reduces to $B=1$ in the time-invariant case. In that case $\tilde{\mathbf{H}}_{i}^{(j)}$ and $\tilde{\boldsymbol{\Lambda}}_{i}^{(j)}$ are diagonal matrices, with the frequency response of $h_{i}^{(j)}[n ; l]$ and $\lambda_{i}^{(j)}[k ; l]$ on the diagonal, respectively.

The way in which we have implemented the multiband OFDM modulator (demodulator) increases in complexity with the number of subbands, because we have a separate upconvertor (downconvertor) and IDFT (DFT) for every subband. We have chosen for this type of implementation because of ease of presentation. However, the proposed modulator (demodulator) can also be implemented using a single upconvertor (downconvertor) and IDFT (DFT), irrespective of the number of subbands. Hence, if we want to compare the complexities of the proposed multiband OFDM system and a single band OFDM system, we only have to focus on the complexities of channel estimation and symbol detection, as will be detailed in the next subsections.

\section{A. Channel Estimation}

In this section, an algorithm is proposed that estimates the acoustic underwater channel for individual OFDM symbols. In other words, only the pilots inside $\mathbf{s}_{i}^{(j)}$ will be used to estimate $\tilde{\Lambda}_{i}^{(j)}$. To this end we exploit the structure that is present in $\tilde{\Lambda}_{i}^{(j)}$, or equivalently the structure that is present in $\boldsymbol{\Lambda}_{i}^{(j)}$. Since $\lambda_{i}^{(j)}[k ; l]$ is the $N / K$-times downsampled version of $c[n] \circledast h_{i}^{(j)}[n ; l] \circledast c[n]$, where $h_{i}^{(j)}[n ; l]$ is a causal time-varying FIR filter with length $L_{h}$, we may assume that $\lambda_{i}^{(j)}[k ; l]$ is a causal time-varying FIR filter with length $L_{\lambda}=\left\lceil\left(L_{h}-1\right) K / N\right\rceil+1$, where we ignore the sidelobes that result from the ideal lowpass filtering with $c[n]$ (carried out twice, once at the transmitter and once at the receiver). Further, instead of modeling $\lambda_{i}^{(j)}[k ; l]$ by means of a timeinvariant channel corrupted by a CFO, as done in [14], we consider a more general framework and model $\lambda_{i}^{(j)}[k ; l]$ by means of a BEM [17], [18]. Specifically, we will model $\boldsymbol{\lambda}_{i}^{(j)}[l]=\left[\lambda_{i}^{(j)}[0 ; l], \ldots, \lambda_{i}^{(j)}[K-1 ; l]\right]^{T}$ as a superposition of $Q$ fixed functions $\mathbf{b}_{q}=\left[b_{q}[0], \ldots, b_{q}[K-1]\right]^{T}$ weighted with $Q$ channel-dependent coefficients $c_{i, q}^{(j)}[l]$, with $Q \ll K$ :

$$
\boldsymbol{\lambda}_{i}^{(j)}[l]=\sum_{q=0}^{Q-1} \mathbf{b}_{q} c_{i, q}^{(j)}[l]=\mathbf{B c}_{i}^{(j)}[l],
$$

where $\mathbf{B}=\left[\mathbf{b}_{0}, \ldots, \mathbf{b}_{Q-1}\right]$ is the BEM functions matrix and $\mathbf{c}_{i}^{(j)}[l]=\left[c_{i, 0}^{(j)}[l], \ldots, c_{i, Q-1}^{(j)}[l]\right]^{T}$ is the BEM coefficient vector related to the $l$-th tap. In this manner the number of unknowns for the $l$-th tap is reduced from $K$ to $Q$. The number of basis functions $Q$ should generally be chosen as the minimum value which results in a reasonably accurate channel model. Values which are too small or too large could result in performance penalties. The actual value of $Q$ will depend on the type of basis functions that are chosen. For the present analysis we use the complex exponential basis functions given by $b_{q}[k]=e^{\iota 2 \pi k[q-(Q-1) / 2] / K_{\mathrm{b}}}$, where $K_{\mathrm{b}}$ is the period of the basis functions generally chosen larger than the OFDM symbol size $K$, e.g., $K_{\mathrm{b}}=2 K$. In that case, a good rule of thumb for the number of basis functions is $Q \approx\left\lceil f_{\mathrm{D}} T N K_{\mathrm{b}} / K\right\rceil$.
To estimate the BEM coefficients, we use the $K_{\mathrm{t}}$ training symbols (pilots) that were inserted in every block of $K_{\mathrm{d}}$ data symbols as discussed in Section III. The pilot scheme we consider here is the one proposed in [18], where every $\mathbf{s}_{p}$ (and thus every OFDM symbol $\mathbf{s}_{i}^{(j)}$ ) consists of $M$ pilot clusters of length $K_{\mathrm{t}} / M$. For the $m$-th pilot cluster, denoted as $\check{\mathbf{s}}_{m}=\left[\mathbf{s}_{i}^{(j)}\right]_{k_{m}: k_{m}+K_{\mathrm{t}} / M-1}$, we then select a related set of received samples $\check{\mathbf{z}}_{i, m}^{(j)}=\left[\mathbf{z}_{i}^{(j)}\right]_{k_{m}+D: k_{m}+K_{\mathrm{t}} / M-D-1}$, where $D$ is a parameter that can be used to control the selected number of output samples. As shown in Appendix D (see also [18]), it is then possible to express $\check{\mathbf{z}}_{i}^{(j)}=\left[\check{\mathbf{z}}_{i, 0}^{(j) T}, \ldots, \check{\mathbf{z}}_{i, M-1}^{(j) T}\right]^{T}$ as

$$
\check{\mathbf{z}}_{i}^{(j)}=\check{\mathbf{D}} \check{\mathbf{S}} \mathbf{c}_{i}^{(j)}+\check{\mathbf{d}}_{i}^{(j)},
$$

where $\mathbf{c}_{i}^{(j)}=\left[\mathbf{c}_{i, 0}^{(j) T}, \ldots, \mathbf{c}_{i, Q-1}^{(j) T}\right]^{T}$ with $\mathbf{c}_{i, q}^{(j)}=$ $\left[c_{i, q}^{(j)}[0], \ldots, c_{i, q}^{(j)}\left[L_{\lambda}-1\right]\right]^{T}$, and where $\check{\mathbf{D}}, \check{\mathbf{S}}$, and $\check{\mathbf{d}}_{i}^{(j)}$ are defined in Appendix D. Note that $\check{\mathbf{D}} \check{\mathrm{S}}$ has size $\left(K_{\mathrm{t}}-2 M D\right) \times L_{\lambda} Q$.

In [18], different methods have been proposed to solve (10) for $\mathbf{c}_{i}^{(j)}$, which is complicated by the fact that the interference term $\check{\mathbf{d}}_{i}^{(j)}$ itself depends on $\mathbf{c}_{i}^{(j)}$. For simplicity reasons, we apply the least-squares (LS) estimator in this work:

$$
\hat{\mathbf{c}}_{i}^{(j)}=(\check{\mathbf{D}} \check{\mathbf{S}})^{\dagger} \check{\mathbf{z}}_{i}^{(j)} .
$$

For more complex estimators, such as the best linear unbiased estimator (BLUE) or the linear minimum mean square error (LMMSE) estimator, we refer the interested reader to [18]. Finally, an estimate for $\tilde{\Lambda}_{i}^{(j)}$ can easily be constructed using (9) and the relation between $\tilde{\Lambda}_{i}^{(j)}$ and $\boldsymbol{\Lambda}_{i}^{(j)}$. The optimal value for $D$ depends on the type of estimator and of course also on the channel. In [18], it is argued that for the LS estimator, $D$ should be chosen as large as possible, i.e., $D=\left(K_{\mathrm{t}} / M-1\right) / 2$, but we find that for highly time-varying underwater channels a somewhat better performance is obtained with $D=0$. Anyway, for time-invariant channels the largest possible $D$ is always optimal, whatever the type of estimator, since there is no ICI.

Under the simplifying assumption that all received samples are used for channel estimation, the estimation complexity is $\mathcal{O}\left(K L_{\lambda}^{2} Q^{2}\right)$ per OFDM symbol, or in total $\mathcal{O}\left(I J K L_{\lambda}^{2} Q^{2}\right)$. For a single band of $J K$ carriers the complexity is $\mathcal{O}\left(I J K J^{2} L_{\lambda}^{2} Q^{2}\right)=\mathcal{O}\left(I J^{3} K L_{\lambda}^{2} Q^{2}\right)$, since the channel length increases by a factor $J$. Hence, the multiband OFDM approach significantly reduces the channel estimation complexity compared to traditional OFDM. In the time-invariant case (or the time-varying case with a single Doppler rate parameter [15]), where we can set $Q=1$ and $D=\left(K_{\mathrm{t}} / M-1\right) / 2$, however, the channel estimation can be simplified due to the specific structure of $\check{\mathbf{D S}}$, as indicated in [14]. The channel estimation complexity is then determined by one transformation from the frequency domain to the time domain and one transformation back. If $L_{\lambda}>\ln (K)$, these transformations can be computed using fast Fourier transforms (FFTs) [14], which amounts to a complexity of $\mathcal{O}(K \ln (K))$ per OFDM symbol, or in total $\mathcal{O}(I J K \ln (K))$. For a single band of $J K$ carriers the complexity becomes 
$\mathcal{O}(I J K \ln (J K))$, which gets close to $\mathcal{O}(I J K \ln (K))$ but only if $J$ is much much smaller than $K$. If on the other hand $L_{\lambda} \leq \ln (K)$, the transformations are more conveniently computed with matrix-vector multiplications. This amounts to a complexity of $\mathcal{O}\left(K L_{\lambda}\right)$ per OFDM symbol, or in total $\mathcal{O}\left(I J K L_{\lambda}\right)$. For a single band of $J K$ carriers the complexity becomes $\mathcal{O}\left(I J K J L_{\lambda}\right)=\mathcal{O}\left(I J^{2} K L_{\lambda}\right)$. So even in this case there is an advantage of the multiband OFDM approach over traditional OFDM, but the advantage is smaller than before and it can be negligible depending on the choice of parameters. Finally, it is remarked that there will always be a small performance loss related to the multiband approach, because we ignore the effect of the sidelobes of the ideal lowpass filtering with $c[n]$.

\section{B. Equalization and Despreading}

In this section dealing with equalization and despreading, we assume that every OFDM symbol $\mathbf{s}_{i}^{(j)}$ or $\mathbf{s}_{p}$, which was assumed to have energy $K$, is white with unit variance

$$
\mathrm{E}\left(\mathbf{s}_{i}^{(j)} \mathbf{s}_{i}^{(j) H}\right)=\mathrm{E}\left(\mathbf{s}_{p} \mathbf{s}_{p}^{H}\right)=\mathbf{I}_{K},
$$

and that the different noise vectors $\boldsymbol{\eta}_{i}^{(j)}$ are white and mutually uncorrelated with different variances

$$
\mathrm{E}\left(\boldsymbol{\eta}_{i}^{(j)} \boldsymbol{\eta}_{i^{\prime}}^{\left(j^{\prime}\right) H}\right)= \begin{cases}\sigma_{i}^{(j) 2} \mathbf{I}_{K}, & \text { if } i=i^{\prime} \text { and } j=j^{\prime} \\ \mathbf{0}_{K \times K}, & \text { otherwise }\end{cases}
$$

The variances $\sigma_{i}^{(j) 2}$ are estimated with the help of null subcarriers in the pilot clusters (see Section V). Instead of following a suboptimal two-step approach of equalization followed by despreading, we here propose a joint approach with improved results. To derive the joint approach, we start by stacking all the $I J / P$ output vectors $\mathbf{z}_{i}^{(j)}$ related to the same input vector $\mathbf{s}_{p}, \mathbf{z}_{p}=\left[\ldots, \mathbf{z}_{i}^{(j) T}, \ldots\right]^{T},(i, j) \in \mathcal{I}_{p}$, leading to

$$
\mathbf{z}_{p}=\tilde{\boldsymbol{\Lambda}}_{p} \mathbf{s}_{p}+\boldsymbol{\eta}_{p}
$$

where $\boldsymbol{\eta}_{p}$ is similarly defined as $\mathbf{z}_{p}$ and $\tilde{\boldsymbol{\Lambda}}_{p}=$ $\left[\ldots, \tilde{\boldsymbol{\Lambda}}_{i}^{(j) T}, \ldots\right]^{T},(i, j) \in \mathcal{I}_{p}$, which is a matrix of size $I J K / P \times K$. Owing to the assumption (13), the covariance matrix of $\boldsymbol{\eta}_{p}$ is given by $\mathbf{R}_{p}=\mathrm{E}\left(\boldsymbol{\eta}_{p} \boldsymbol{\eta}_{p}^{H}\right)=$ $\operatorname{diag}\left(\ldots, \sigma_{i}^{(j) 2} \mathbf{I}_{K}, \ldots\right),(i, j) \in \mathcal{I}_{p}$. From (14), we can then estimate $\mathbf{s}_{p}$ by linear processing. Adopting an LMMSE estimator, we obtain

$$
\begin{aligned}
\hat{\mathbf{s}}_{p}= & \hat{\tilde{\mathbf{\Lambda}}}_{p}^{H}\left(\hat{\tilde{\mathbf{\Lambda}}}_{p} \hat{\tilde{\mathbf{\Lambda}}}_{p}^{H}+\hat{\mathbf{R}}_{p}\right)^{-1} \mathbf{z}_{p} \\
= & \left(\hat{\tilde{\mathbf{\Lambda}}}_{p}^{H} \hat{\mathbf{R}}_{p}^{-1} \hat{\tilde{\mathbf{\Lambda}}}_{p}+\mathbf{I}_{K}\right)^{-1} \hat{\tilde{\mathbf{\Lambda}}}_{p}^{H} \hat{\mathbf{R}}_{p}^{-1} \mathbf{z}_{p} \\
= & \left(\sum_{(i, j) \in \mathcal{I}_{p}} \hat{\sigma}_{i}^{(j)-2} \hat{\tilde{\boldsymbol{\Lambda}}}_{i}^{(j) H} \hat{\tilde{\boldsymbol{\Lambda}}}_{i}^{(j)}+\mathbf{I}_{K}\right)^{-1} \\
& \times \sum_{(i, j) \in \mathcal{I}_{p}} \hat{\sigma}_{i}^{(j)-2} \hat{\tilde{\mathbf{\Lambda}}}_{i}^{(j) H} \mathbf{z}_{i}^{(j)},
\end{aligned}
$$

where $\hat{\tilde{\mathbf{\Lambda}}}_{p}, \hat{\tilde{\boldsymbol{\Lambda}}}_{i}^{(j)}, \hat{\mathbf{R}}_{p}$, and $\hat{\sigma}_{i}^{(j)}$ represent the estimated versions of $\tilde{\mathbf{\Lambda}}_{p}, \tilde{\boldsymbol{\Lambda}}_{i}^{(j)}, \mathbf{R}_{p}$, and $\sigma_{i}^{(j)}$.
Note that the complexity of the joint equalization and despreading operation is $\mathcal{O}\left(K^{3}\right)$ per data symbol vector, or in total $\mathcal{O}\left(P K^{3}\right)$. For a single band of $J K$ carriers this complexity is $\mathcal{O}\left(P / J J^{3} K^{3}\right)=\mathcal{O}\left(P J^{2} K^{3}\right)$, since the number of data symbol vectors decreases by a factor $J$. Hence, the multiband OFDM approach also significantly reduces the complexity of the joint equalization and despreading operation compared to traditional OFDM. However, this is not true when the circularly banded nature of $\tilde{\Lambda}_{i}^{(j)}$ is taken into account [8][11]. Remember that $\tilde{\Lambda}_{i}^{(j)}$ can be assumed to have a bandwidth $B$, which in the time-invariant case (or the time-varying case with a single Doppler rate parameter [15]) reduces to $B=1$ when $\tilde{\Lambda}_{i}^{(j)}$ is diagonal. For this case, the complexity of the joint equalization and despreading operation is reduced to $\mathcal{O}\left(K B^{2}\right)$ per data symbol vector, or in total $\mathcal{O}\left(P K B^{2}\right)$, which equals the complexity of a single band with $J K$ carriers.

\section{Turbo Decoding}

A standard turbo decoder is used to decode the encoded bits [20]. At the input it requires the log-likelihood ratios (LLRs) of the encoded bits after equalization, and the output consists of the estimated information bits before encoding. The question that remains is how we compute the a priori LLRs of the encoded bits after equalization. First, we need to derive the probability density function (PDF) of the estimated data symbols $s_{p}[k]=\left[\mathbf{s}_{p}\right]_{k}$ given that the related transmitted data symbol was $\alpha$. The exact derivation of this conditional PDF is involved, but we may approximate it as Gaussian with mean $\mu_{p, \alpha}[k]$ and variance $\sigma_{p, \alpha}^{2}[k]$, a common procedure in turbo equalization [21]. Specifically, the mean $\mu_{p, \alpha}[k]$ and variance $\sigma_{p, \alpha}^{2}[k]$ can be derived from [21] as

$$
\begin{aligned}
\mu_{p, \alpha}[k] & =t_{p}[k] \alpha \\
\sigma_{p, \alpha}^{2}[k] & =t_{p}[k]\left(1-t_{p}[k]\right),
\end{aligned}
$$

where

$$
\begin{aligned}
& t_{p}[k]= {\left[\hat{\tilde{\mathbf{\Lambda}}}_{p}^{H}\left(\hat{\tilde{\mathbf{\Lambda}}}_{p} \hat{\tilde{\mathbf{\Lambda}}}_{p}^{H}+\hat{\mathbf{R}}_{p}\right)^{-1} \hat{\tilde{\mathbf{\Lambda}}}_{p}\right]_{k, k} } \\
&= {\left[\left(\hat{\tilde{\mathbf{\Lambda}}}_{p}^{H} \hat{\mathbf{R}}_{p}^{-1} \hat{\tilde{\mathbf{\Lambda}}}_{p}+\mathbf{I}_{K}\right)^{-1} \hat{\tilde{\mathbf{\Lambda}}}_{p}^{H} \hat{\mathbf{R}}_{p}^{-1} \hat{\tilde{\mathbf{\Lambda}}}_{p}\right]_{k, k} } \\
&= {\left[\left(\sum_{(i, j) \in \mathcal{I}_{p}} \hat{\sigma}_{i}^{(j)-2} \hat{\tilde{\mathbf{\Lambda}}}_{i}^{(j) H} \hat{\tilde{\mathbf{\Lambda}}}_{i}^{(j)}+\mathbf{I}_{K}\right)^{-1}\right.} \\
&\left.\times \sum_{(i, j) \in \mathcal{I}_{p}} \hat{\sigma}_{i}^{(j)-2} \hat{\tilde{\mathbf{\Lambda}}}_{i}^{(j) H} \hat{\tilde{\mathbf{\Lambda}}}_{i}^{(j)}\right]_{k, k} .
\end{aligned}
$$

Using these formulations for $\mu_{p, \alpha}[k]$ and $\sigma_{p, \alpha}^{2}[k]$, one can then compute the LLRs of the encoded bits after equalization for different PSK alphabets as indicated in [21].

Note that channel estimation, equalization and despreading, as well as turbo decoding can also run in an iterative loop, where in each step the performance is improved, similar to [21]-[24]. However, for complexity reasons we do not consider such an iterative framework for the proposed acoustic communication system. 


\section{Signal Parameters}

The multiband OFDM system described in the above sections was tested during sea experiments. The objective was to communicate over long distances in an inconspicuous manner, i.e., to set up a communication link at as low as possible an SNR. Two target data rates were specified at values of 4.2 and $75 \mathrm{bit} / \mathrm{s}$ in an available bandwidth of $3.6 \mathrm{kHz}$. These rates are henceforth referred to as $\mathrm{R} 1$ and $\mathrm{R} 2$, respectively.

A maximal-length sequence of length 4095 is used for the preamble, with a theoretical processing gain of $36 \mathrm{~dB}$. This sequence is modulated on sinc pulses with a bandwidth of $3600 \mathrm{~Hz}$ at a chip rate of $3600 \mathrm{~Hz}$. The sinc pulses are truncated at a generous 100 chip periods at either side, and a guard time of $200 \mathrm{~ms}$ is inserted between the acquisition signal and the communication signal. The use of sinc pulses is motivated by the PAPR and spectral envelope of the preamble, which compare well to those of the covert OFDM signal. The Doppler bank for detection and synchronization is filled with 81 Doppler replicas between -4.8 and $+4.8 \mathrm{~m} / \mathrm{s}$ in $0.12 \mathrm{~m} / \mathrm{s}$ steps.

A CP of $N_{\mathrm{cp}} T=150 \mathrm{~ms}$ is employed to cope with the expected delay spread of the littoral trial areas. The redundancy introduced by the $\mathrm{CP}$ is limited by adopting a substantially longer OFDM symbol with a duration of $N T=1.2 \mathrm{~s}$ and a carrier spacing of $1 /(N T)=1 / 1.2 \mathrm{~Hz}$. As a result, we could pack 4320 carriers into the available bandwidth. As already indicated earlier, OFDM symbols with that many carriers lead to considerable channel estimation and symbol detection costs. That is why we decided to split the band into $J=16$ subbands of $K=256$ carriers each, with $G=14$ guard carriers in between the subbands. The guard bands allow for a Doppler spread of $G /(N T)=14 / 1.2 \approx 12 \mathrm{~Hz}$ between the OFDM subbands, which is much more than the Doppler spread that was encountered at sea. The timing offsets are chosen as $N_{j} T=j 2 \mathrm{~ms}$, and the subband carrier frequencies $f_{j}$ are offset from the overall center frequency $f_{\mathrm{c}}$ according to $f_{j}=f_{\mathrm{c}}+(j-7.5)(K+G) /(N T)=f_{\mathrm{c}}+(j-7.5) \times 225 \mathrm{~Hz}$. The value of $f_{c}$ itself depends on the type of transducer that was used, as explained in the next section. Note that we do not detail the exact value of the rate $1 / T$, since it has a negligible influence on the performance as long as it is larger than the signal bandwidth.

For the pilot design, we use $K_{\mathrm{t}}=160$ pilots per data vector $\mathbf{s}_{p}$ divided into $M=32$ pilot clusters of length $K_{\mathrm{t}} / M=5$. Hence, the number of data symbols per data vector is $K_{\mathrm{d}}=K-K_{\mathrm{t}}=96$. Every cluster is designed according to [25], which is a generalization of the cluster design proposed in [26]. Basically, we put a single nonzero pilot in the middle and surround it by zeros. To improve the efficiency at low SNR, the available power is equally distributed among data symbols and pilots, as indicated in [26]. Since the overall energy of $\mathbf{s}_{p}$ is set to $K$, the nonzero pilots have an energy $K /(2 M)=4$, versus an energy $K /\left(2 K_{\mathrm{d}}\right)=4 / 3$ for the data carriers.

To shift the working regime further to low SNR, we employ a turbo code of rate $1 / 3$ and we also spread the data vectors $\mathbf{s}_{p}$ over a total of $I$ OFDM symbols and $J=16$ subbands using a QPSK constellation. Standard parallel turbo encoders are considered with generator polynomials $(15,11)$ and $(15,13)$ for $\mathrm{R} 1$ and $\mathrm{R} 2$, respectively (in $(a, b), a$ stands for the parity connections in decimal form, whereas $b$ stands for the feedback connections in decimal form). The interleavers have lengths of 128 and 640 bits, which correspond to 125 and 637 information bits as the three tail bits are reserved to terminate the first constituent encoder. R1 carries 125 information bits in $P=2$ turbo-encoded data vectors with $I=21$ OFDM symbols per subband. The vectors are distributed over $I J=336$ slots, time-frequency wise in a checkerboard fashion, at a data rate of $125 /(21 \times 1.35) \approx 4.4 \mathrm{bit} / \mathrm{s}$. R2 carries three code blocks with a total of $3 \times 637=1911$ information bits in $P=30$ turbo-encoded data vectors, using $I=17$ OFDM symbols per subband. These vectors are spread out over $I J=272$ slots at a data rate of $1911 /(17 \times 1.35) \approx 83 \mathrm{bit} / \mathrm{s}$. Each vector is repeated in time and in frequency, with the precise distribution being too cumbersome to detail. The effective data rates are $R_{1}=4.2 \mathrm{bit} / \mathrm{s}$ and $R_{2}=78 \mathrm{bit} / \mathrm{s}$ if the overhead of the preamble is taken into account.

\section{EXPERIMENTS AND RESULTS}

\section{A. Sea Trials}

The waveforms described in Section $\mathrm{V}$ were broadcast and recorded during sea trials in northern Europe, in $\mathrm{Au}-$ gust/September 2007. Three experiments are considered here with various degrees of delay and Doppler spreading. Fig. 5 presents an area map and Fig. 6 measured power delay profiles and Doppler spectra for the three cases. Two experiments are analyzed for site A in the Baltic Sea. Waveforms were transmitted with a prototype acoustic modem, which was suspended in the water column from a stationary surface ship, which is to say, one that used a dynamic positioning system to stay at a specified position. At the other end of the channel the signals were recorded on a vertical hydrophone chain, deployed from an anchored ship. The modem was deployed in a submerged sound channel whose axis was $40 \mathrm{~m}$ below the surface. A single hydrophone at a 50-m depth is used for the analysis, which is within the sound channel. Typical impulse responses in the Baltic sound channel display a crescendo of multipath arrivals, with an overall time spread that increases with range. The distance is in fact the main difference between the two experiments at site A, which were executed at ranges of 8 and $52 \mathrm{~km}$. Both channels feature a small Doppler spread, which is due to the stationary geometry and the absence of surface interactions of sound trapped in the channel. The OFDM signals were transmitted every six minutes as part of a periodic transmit schedule comprising various covert modulations and a probe signal [28]. With each cycle the OFDM source level was reduced by $2 \mathrm{~dB}$. The center frequency of the signals transmitted with the modem is $f_{\mathrm{c}}=3.3 \mathrm{kHz}$.

The third channel in Fig. 6 is a snapshot from an experiment on the Norwegian continental shelf. A different transducer was used, transmitting the same OFDM waveforms as the abovementioned modem, but at a center frequency of $f_{\mathrm{c}}=5.0 \mathrm{kHz}$ and at an interval of eight minutes. The source was towed at a nominal depth of $60 \mathrm{~m}$ by a surface ship. It sailed away from the anchored receiver platform at a speed of 


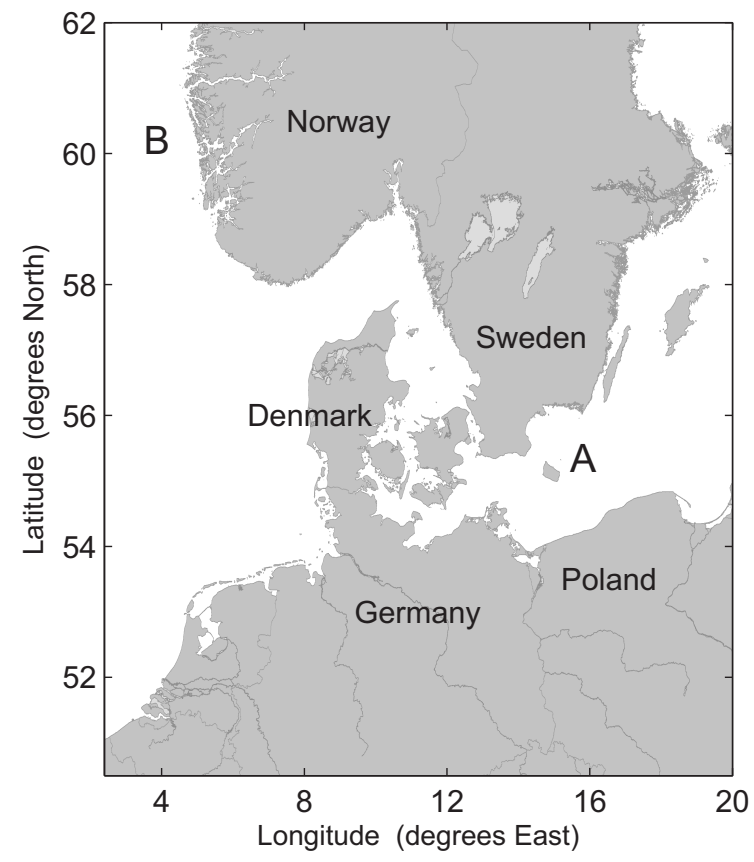

Fig. 5. Area map of the UCAC sea trials.

$\approx 2.5 \mathrm{~m} / \mathrm{s}$, starting nearby and reaching a distance of $38 \mathrm{~km}$. The overall downward refracting sound speed profile favored deeper hydrophones of the receiver chain over shallow ones, with a hydrophone at $90 \mathrm{~m}$ being selected for analysis. During this experiment the source level was constant, and the SNR at reception decreased with time because of the increasing range. The representative channel in Fig. 6 reveals a relatively sparse pattern of multipath arrivals, quite different from the Baltic impulse responses, and a considerable Doppler spread. The measured Doppler variance is partly due to a timevariable channel (multipath fading), and partly to a timevariable Doppler shift introduced by motion of the transmitter ship on a rough sea surface.

\section{B. Results}

Results for the channels of area A are shown in Fig. 7. The figure gives the number of bit errors, after the turbo decoder, for an SNR that decreases with the cycle counter. These SNRs are estimated with the method detailed in [28], and have an uncertainty of $2 \mathrm{~dB}$. Exhibiting little delay and Doppler spreading, the channel at $8 \mathrm{~km}$ is benign. Fig. 7 shows that $\mathrm{R} 2$ is successfully demodulated down to an SNR of $-9 \mathrm{~dB}$, whereas R1 is correctly received down to the lowest realized SNR of $-17 \mathrm{~dB}$. The delay spread is longer at $52 \mathrm{~km}$ and appears to influence the receiver performance, with the SNR required for demodulation being a few $\mathrm{dB}$ higher than at $8 \mathrm{~km}$. However, at $4.2 \mathrm{bit} / \mathrm{s}$ the performance is not limited by the OFDM receiver, but by failure of detection. With the signal energy spread over a large number of multipath arrivals, the detection threshold is not met at SNRs for which a monopath channel would still allow detection. The critical SNR for the R1 demodulator itself is not revealed by Fig. 7. It is possible to synchronize by brute force, where we step through the recorded data in 10-ms steps and compute the BER for each starting point. This procedure reveals that the SNR limit of R1
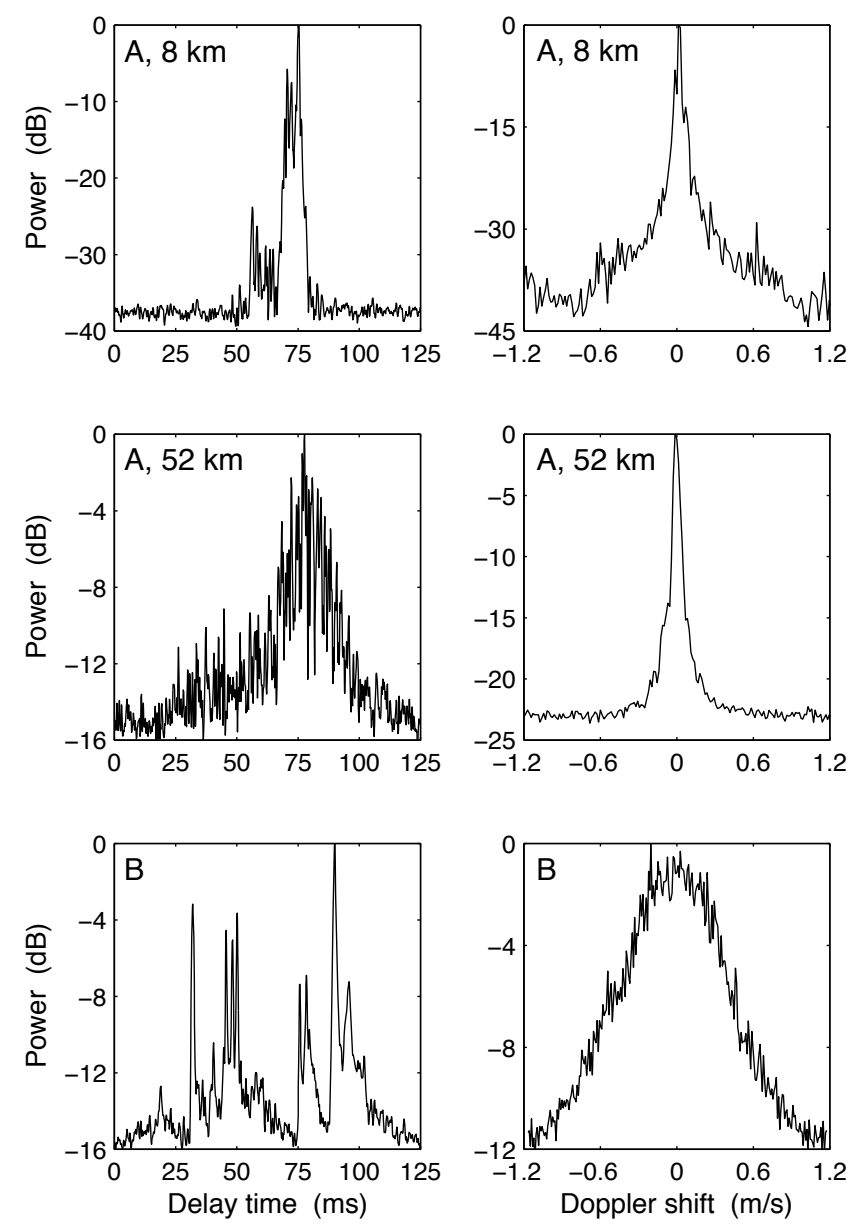

Fig. 6. Power delay profiles and Doppler power spectra for the case studies. The graphs reflect 32-s averages measured with the probe signal and processing described in [27].

is about $-18 \mathrm{~dB}$ for the Baltic sound channel. For example, one more zero can be obtained at $52-\mathrm{km}$ (the ninth cycle in Fig. 7), and comparable SNR limits are found for different hydrophone channels and experiments (not shown).

The experiment at site B (Fig. 8) is characterized by a high time variability. The delay spread shown in Fig. 6 decreases somewhat with range, in contrast to the Baltic sound channel, but the Doppler spread is large throughout. The experiment is further characterized by awkward crunch and click sounds produced onboard the receiver platform. Noise is particularly loud when the range is $20 \mathrm{~km}$, causing a big dip in the SNR and an isolated R2 failure. Otherwise the bit errors occur at distances beyond $30 \mathrm{~km}$. R2 is successful at SNRs down to approximately $-7 \mathrm{~dB}$. No bit errors occur at $4.2 \mathrm{bit} / \mathrm{s}$, for which the SNR is $-13 \mathrm{~dB}$ at the longest achieved range. However, demodulation of other, more difficult (i.e. lower SNR) hydrophone channels reveals that $-13 \mathrm{~dB}$ is more or less the performance limit for R1 in the North Sea. It appears that the problem is again one of detection rather than communication. It is not possible to plot these results in the fashion of Fig. 8, because the SNR is too low to allow a reliable estimate. However, the detection problem is manifest. For example, if a hydrophone at a depth of $50 \mathrm{~m}$ is used to demodulate R1, the results comprise 8 detection failures, 15 zero-BER receptions, and nothing in between. Compared with 


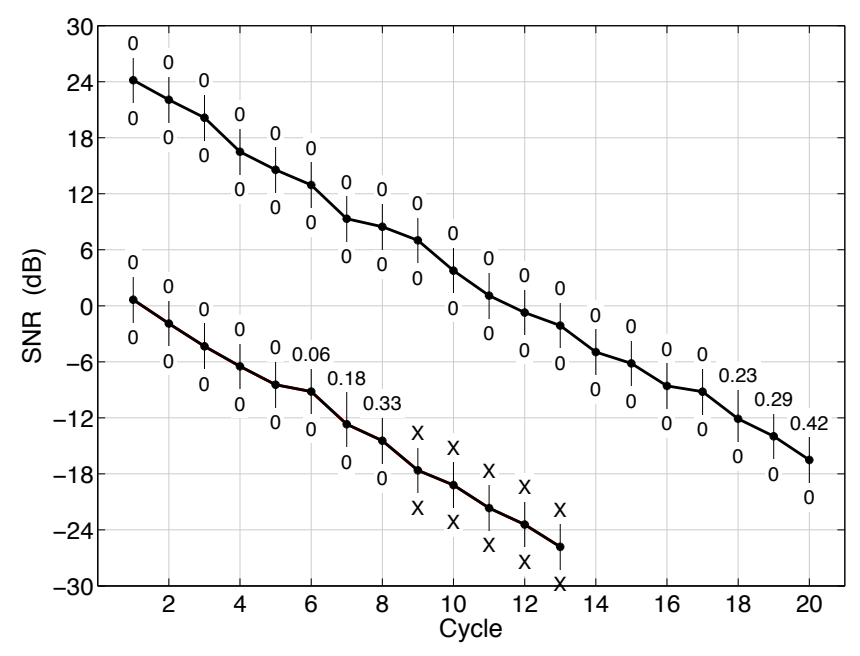

Fig. 7. SNRs for area A, at ranges of $8 \mathrm{~km}$ (upper curve) and $52 \mathrm{~km}$ (lower curve), versus the cycle counter. The BER is plotted for each reception of R1 and R2, respectively below and above the curves. Crosses indicate failure of detection $\left(Q=1, D=2, L_{\lambda}=8\right)$.

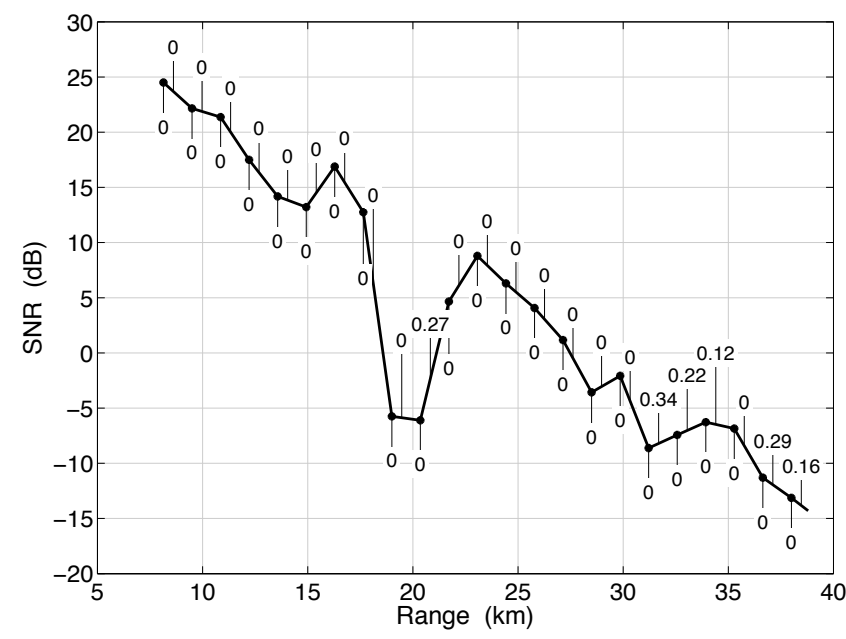

Fig. 8. SNR for the dynamic run at site B. The BERs for R1 and R2 are respectively indicated below and above the curve $\left(Q=3, D=0, L_{\lambda}=8\right)$.

the experiments at site A, signal detection is further hindered by the large Doppler spread which affects the gain of the filter matched to the Doppler-sensitive preamble.

\section{Discussion}

At the lowest achieved SNRs, the covert OFDM signals are inaudible to the ear and invisible to the eye in spectrograms, even when their position in the recorded data is known to the audience. If communication links are to be established at still lower SNRs, the first concern is signal detection and synchronization. Indeed, the limits of the R1 receiver were not exposed during the sea experiments, with a performance limited by detection rather than the OFDM scheme itself. For scenarios with a small Doppler spread this can be solved by using still longer $\mathrm{m}$-sequences for the preamble. Unfortunately this is no option for the dynamic scenario of site B, for which the large Doppler variance destroys the gain of the matched filter.

For Fig. 7, we use the time-invariant estimator with $Q=1$ and $D=2$. For Fig. 8 , we use the time-varying channel

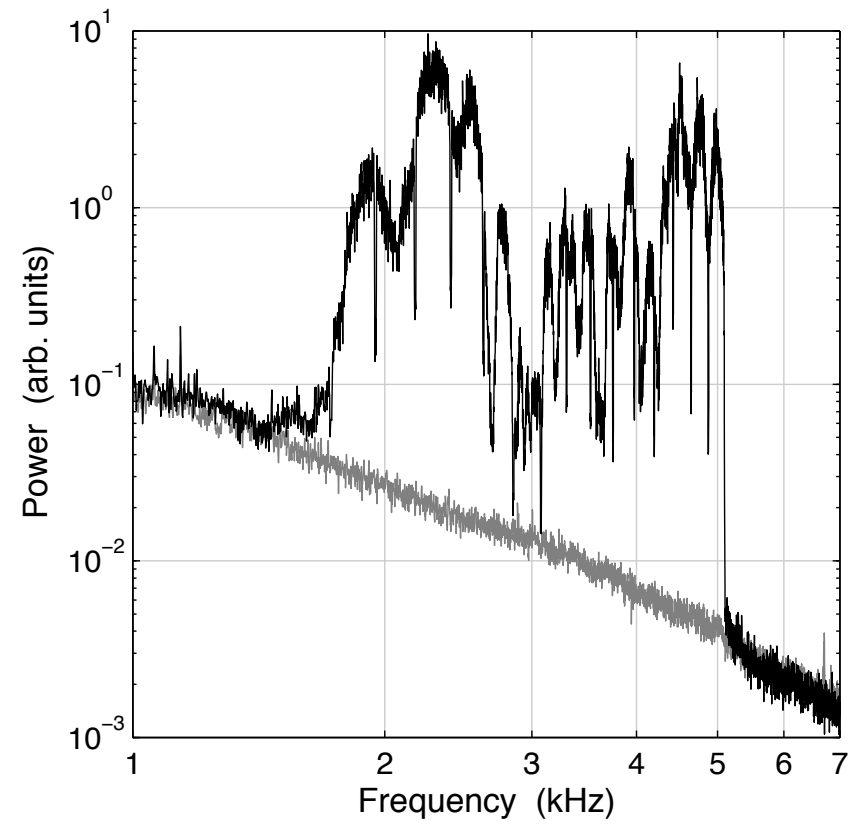

Fig. 9. Power spectral densities of two recordings separated by a 38-dB source level difference.

estimator relying on a BEM consisting of complex exponential basis functions with period $K_{\mathrm{b}}=2 K$. The number of basis functions is set according to $Q \approx\left\lceil f_{\mathrm{D}} T N K_{\mathrm{b}} / K\right\rceil=$ $\left\lceil 2 f_{\mathrm{D}} T N\right\rceil$ (see Section IV-A). For a symbol duration of $N T=1.2 \mathrm{~s}$ and a maximal Doppler spread of $f_{\mathrm{D}} \approx 1 \mathrm{~Hz}$, this leads to $Q=3$. As already indicated in Section IV-A, since $D=0$ leads to better results than $D=2$ for this scenario, we select $D=0$ for this case. The number of channel taps $L_{\lambda}$ is roughly tuned to the time spread of the main multipath arrivals and is set to $L_{\lambda}=8$. Remember that the delay parameter is automatically set to center the chief multipath arrival (of the correlation filter output) in the channel impulse response. This synchronization is performed only once, at the start of the incoming OFDM waveform. Uncertainties in the initial Doppler estimate, and nonconstancy of platform velocities during the 25-30 s duration of the signals, easily cause synchronization errors of a few milliseconds for symbols toward the end of the signal. However, so long as this offset is substantially shorter than the equalizer span it will not cause major problems. No problems are expected for the intended application with a UUV sailing a stable course at some distance from the mother ship. In fact, UUVs tend to sail more stable courses than the surface ships used during the 2007 sea trials.

The choice for the time-invariant estimator for site A is prompted by the small Doppler spread. Application of the time-varying estimator-all else equal—leads to slightly worse results, whereas application of the time-invariant estimator to site B leads to considerably worse results. For example, it gives rise to five additional $\mathrm{R} 2$ receptions with nonzero BER, with bit errors now occurring also at an SNR of $+20 \mathrm{~dB}$.

The assumption (13) that the noise vectors $\boldsymbol{\eta}_{i}^{(j)}$ are white is reasonable for narrow subbands, but their variances differ considerably between subbands. Fig. 9 exhibits the power 


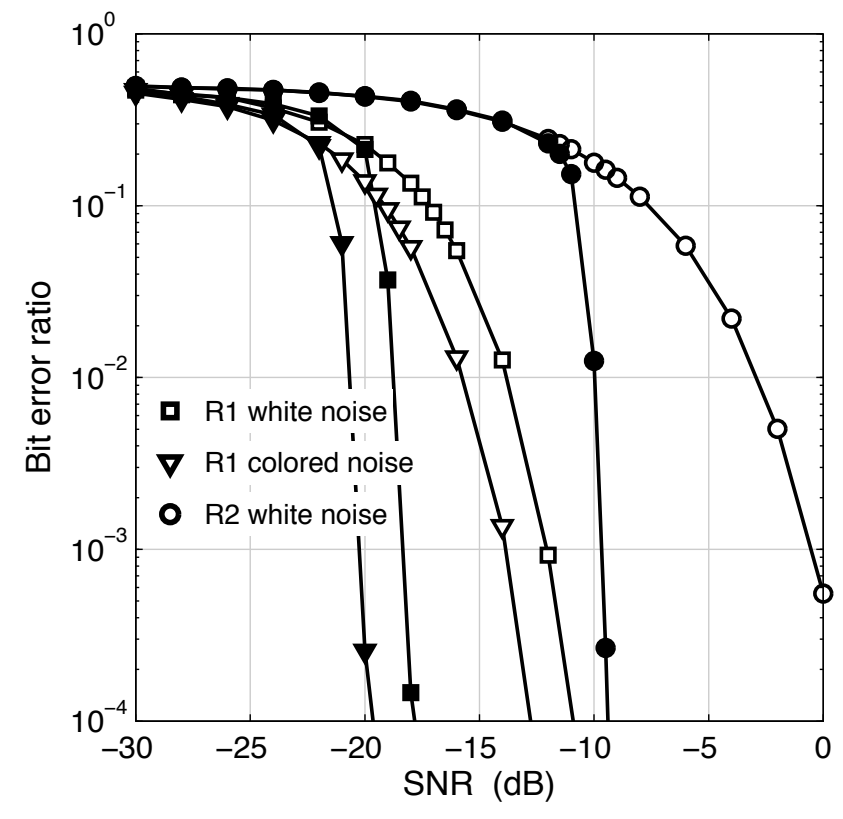

Fig. 10. BER curves for synthetic white noise, and colored noise falling off with $-6 \mathrm{~dB}$ per octave. Both the coded BER (black markers) and uncoded BER (white markers) are given. Proper synchronization is enforced.

spectral density of two recordings from the Baltic Sea, two hours and $38 \mathrm{~dB}$ apart. The spectrum of the recording from the last cycle of the experiment is indistinguishable from that of noise alone, but the contained signal can still be decoded. Notice that the noise power falls off with the frequency, and that the SNR of the received OFDM signals depends on the subband under consideration. The figure also illustrates the distortion of the signal spectrum due to multipath propagation, which gives rise to frequency-selective fading.

A performance benchmark is obtained by adding synthetic noise to the signals delivered by the transmitter, and by feeding the noisy signals to the OFDM receiver. Fig. 10 shows BER curves resulting from thousands of realizations. The curves are shown for white noise added to R1 and R2, and for the coded and uncoded bits. The effect of colored noise is additionally shown for R1. A noise spectrum with a falloff of $6 \mathrm{~dB}$ per octave is used, similar to the noise floor in Fig. 9. Detection is skipped and synchronization enforced in the receiver, and the signals are further characterized by the absence of time or Doppler spreading. The time-invariant estimator is used here with $Q=1, D=2$, and $L_{\lambda}=4$. Note that the SNR in Fig. 10 is exact and defined as the ratio of the signal energy to the noise energy in the rectangular time-frequency window of the signal. Relevant observations are that the uncoded BERs for R1 and R2 are approximately $12 \mathrm{~dB}$ apart, which is in agreement with the ratio of the data rates. However, the difference between the coded BERs is smaller at $9 \mathrm{~dB}$. One possible reason is that the effectiveness of turbo codes increases with the interleaver length, which gives R2 a decoding advantage over R1. Another conclusion from Fig. 9 is that, at a given overall SNR, the noise spectral distribution has an influence on the BER. The colored noise, $-6 \mathrm{~dB}$ per octave in this simulation, offers an advantage of $2 \mathrm{~dB}$ compared with white noise.
Taking the effect of noise color into account, we find errorfree receptions regularly down to $-21 \mathrm{~dB}(\mathrm{R} 1)$ and $-12 \mathrm{~dB}$ (R2) for the simulated data. This is obviously better than the at-sea results, but the differences are not huge. The best at-sea results approach the benchmark values by $\approx 3 \mathrm{~dB}$. The greatest departure is $\approx 8 \mathrm{~dB}$ for $\mathrm{R} 2$ on the Norwegian continental shelf, although this is really due to failure of detection. However, if synchronization is achieved by brute force, we normally find that the limit of the $\mathrm{R} 1$ receiver is only $2-3 \mathrm{~dB}$ better than the detection limit. On average the receiver performance is some $6 \mathrm{~dB}$ below the benchmark.

\section{CONCLUDING REMARKS}

A multiband OFDM modulation is presented for underwater communications at low SNR. Compared with a single-band OFDM system, the multiband approach with joint equalization and despreading leads to a significant reduction of the receiver complexity. The algorithms were tested during sea trials in littoral waters under different conditions of time and Doppler spreading. At $78 \mathrm{bit} / \mathrm{s}$, the SNR limits of the proposed OFDM scheme are clearly revealed. At $4.2 \mathrm{bit} / \mathrm{s}$, message recovery is possible down to an SNR of $-17 \mathrm{~dB}$ in a benign acoustic channel, and down to approximately $-13 \mathrm{~dB}$ in a severely doubly spread channel. However, in all examined cases the system performance at $4.2 \mathrm{bit} / \mathrm{s}$ is not limited by the demodulator, but by failure of detection and initial synchronization. The proposed OFDM communication scheme has been implemented in two prototype acoustic modems, and 2-way autonomous communication has been established between a sailing UUV and a mother ship during integration tests in a Norwegian fjord. Covert operation is to be demonstrated during a final sea trial in September 2008.

\section{ACKNOWLEDGMENT}

The authors are indebted to the entire UCAC consortium and management group. Credit is also due to Jeroen Boschma, Hans Gerritsen, Claudio Fanciullacci, and Paolo Tusoni.

\section{APPENDIX A}

In this appendix, we will show that (2) actually corresponds to transforming $\mathbf{s}_{i}^{(j)}$ by means of a $K$-point unitary IDFT, then upsampling it with a factor $N / K$, and finally circularly lowpass filtering it with a unit-energy filter. To see this, let us rewrite (2) as

$$
\begin{aligned}
\mathbf{x}_{i}^{(j)} & =\mathbf{F}_{N}^{H} \mathbf{T} \mathbf{s}_{i}^{(j)} \\
& =\mathbf{F}_{N}^{H} \mathbf{T} \mathbf{F}_{K} \mathbf{F}_{K}^{H} \mathbf{s}_{i}^{(j)} \\
& =\sqrt{\frac{N}{K}} \mathbf{F}_{N}^{H} \mathbf{T} \mathbf{T}^{T} \mathbf{F}_{N} \mathbf{P} \mathbf{F}_{K}^{H} \mathbf{s}_{i}^{(j)} \\
& =\mathbf{F}_{N}^{H} \tilde{\mathbf{C}} \mathbf{F}_{N} \mathbf{P} \mathbf{F}_{K}^{H} \mathbf{s}_{i}^{(j)} \\
& =\mathbf{C P F}_{K}^{H} \mathbf{s}_{i}^{(j)}
\end{aligned}
$$

where $\mathbf{P}$ is the $N \times K$ upsampling matrix given by $\mathbf{P}=$ $\mathbf{I}_{K} \otimes\left[1, \mathbf{0}_{1, N / K-1}\right]^{T}, \tilde{\mathbf{C}}$ is the $N \times N$ diagonal matrix given by $[\tilde{\mathbf{C}}]_{n, n}=\tilde{c}[n]$ with $\tilde{c}[n]=\sqrt{N} / \sqrt{K}$ for $n=$ $0, \ldots, K / 2-1, N-K / 2, \ldots, N-1$ and $\tilde{c}[n]=0$ for 
$n=K / 2, \ldots, N-K / 2-1$, and $\mathbf{C}$ is the $N \times N$ circular matrix given by $[\mathbf{C}]_{n, n^{\prime}}=c\left[\left(n-n^{\prime}\right) \bmod N\right]$, with $c[n]=1 / \sqrt{K N} \exp (-\iota \pi n / N) \sin (\pi n K / N) / \sin (\pi n / N)$ for $n=0,1, \ldots, N-1$, and where we have made use of the property $\sqrt{N} / \sqrt{K} \mathbf{T}^{T} \mathbf{F}_{N} \mathbf{P}=\mathbf{F}_{K}$. The unit-energy circular lowpass filter we thus need to represent (2) is $c[n]$ with frequency response $\tilde{c}[n]$. This concludes the proof.

\section{APPENDIX B}

If i) the CP length $N_{\mathrm{cp}}$ is large enough for the channel (including all delay effects) to fit within the $\mathrm{CP}$ for every subband, i.e., $N_{\mathrm{cp}} \geq L_{h}-1+\max _{j, j^{\prime}}\left(N_{j}-N_{j^{\prime}}\right)$, then $\mathbf{y}_{i}^{(j)}$ only depends on the $i$-th OFDM symbol of all subbands, i.e., $\mathbf{x}_{i}^{\left(j^{\prime}\right)}$ for $j^{\prime}=1, \ldots, J$, and the relationship is given by

$$
\mathbf{y}_{i}^{(j)}=\sum_{j^{\prime}=0}^{J-1} \mathbf{H}_{i}^{\left(j, j^{\prime}\right)} \mathbf{x}_{i}^{\left(j^{\prime}\right)}+\boldsymbol{\zeta}_{i}^{(j)},
$$

where $\boldsymbol{\zeta}_{i}^{(j)}$ is similar defined from $\bar{\varepsilon}[n]$ as $\mathbf{y}_{i}^{(j)}$ from $\bar{v}[n]$, and where $\mathbf{H}_{i}^{\left(j, j^{\prime}\right)}$ is the $N \times N$ channel matrix given by $\left[\mathbf{H}_{i}^{\left(j, j^{\prime}\right)}\right]_{n, n^{\prime}}$ $\bar{h}\left[n_{i}^{(j)}+N_{\mathrm{cp}}+n ;\left(N_{j}+n-N_{j^{\prime}}-n^{\prime}\right) \bmod N\right]^{n}$ $\exp \left\{-\iota 2 \pi\left[f_{j}\left(n_{i}^{(j)}+N_{\mathrm{cp}}+n\right)-f_{j^{\prime}}\left(n_{i}^{\left(j^{\prime}\right)}+N_{\mathrm{cp}}+n^{\prime}\right)\right] T\right\}$. If we further assume that ii) the different carriers are separated by $(K+G) /(N T)$, where $1 /(N T)$ is the carrier spacing and $G /(N T)$ is some frequency guard between the subbands, then we can write $f_{j^{\prime}}$ as $f_{j^{\prime}}=f_{j}+\left(j^{\prime}-j\right)(K+G) /(N T)$ and $\left[\mathbf{H}_{i}^{\left(j, j^{\prime}\right)}\right]_{n, n^{\prime}}=$ $\bar{h}\left[n_{i}^{(j)}+N_{\mathrm{cp}}+n ;\left(N_{j}+n-N_{j^{\prime}}-n^{\prime}\right) \bmod N\right]$ $\exp \left[-\iota 2 \pi f_{j}\left(N_{j}+n-N_{j^{\prime}}-n^{\prime}\right) T\right]$ $\exp \left[\iota 2 \pi\left(j^{\prime}-j\right)(K+G)\left(n_{i}^{\left(j^{\prime}\right)}+N_{\mathrm{cp}}+n^{\prime}\right) / N\right]$. Upon moving this last exponential factor from $\mathbf{H}_{i}^{\left(j, j^{\prime}\right)}$ to $\mathbf{x}_{i}^{\left(j^{\prime}\right)}$, can be rewritten as

$$
\mathbf{y}_{i}^{(j)}=\mathbf{H}_{i}^{(j)} \mathbf{x}_{i}^{(j)}+\sum_{\substack{j^{\prime}=0 \\ j^{\prime} \neq j}}^{J-1} \mathbf{H}_{i, \text { new }}^{\left(j, j^{\prime}\right)} \mathbf{x}_{i, \text { new }}^{\left(j, j^{\prime}\right)}+\boldsymbol{\zeta}_{i}^{(j)},
$$

where $\mathbf{H}_{i}^{(j)}=\mathbf{H}_{i}^{(j, j)}$ is the $N \times N$ quasi-circular channel matrix of interest given by $\left[\mathbf{H}_{i}^{(j)}\right]_{n, n^{\prime}}=h_{i}^{(j)}\left[n ;\left(n-n^{\prime}\right) \bmod N\right]$ with $h_{i}^{(j)}[n ; l]=\bar{h}\left[n_{i}^{(j)}+N_{\mathrm{cp}}+n ; l\right] \exp \left(-\iota 2 \pi f_{j} l T\right)$, $\mathbf{H}_{i, \text { new }}^{\left(j, j^{\prime}\right)}$ is the $N \times N$ quasi-circular channel matrix given by $\left[\mathbf{H}_{i, \text { new }}^{\left(j, j^{\prime}\right)}\right]_{n, n^{\prime}}=h_{i, \text { new }}^{\left(j, j^{\prime}\right)}\left[n ;\left(n-n^{\prime}\right) \bmod N\right]$ with $h_{i, \text { new }}^{\left(j, j^{\prime}\right)}[n ; l]=\bar{h}\left[n_{i}^{(j)}+N_{\mathrm{cp}}+n ; N_{j}-N_{j^{\prime}}+l\right]$ $\exp \left[-\iota 2 \pi f_{j}\left(N_{j}-N_{j^{\prime}}+l\right) T\right], \quad$ and $\quad \mathbf{x}_{i, \text { new }}^{\left(j, j^{\prime}\right)}$ the $N \times 1$ vector given by $\left[\mathbf{x}_{i, \text { new }}^{\left(j, j^{\prime}\right)}\right]_{n}=$ $\exp \left[\iota 2 \pi\left(j^{\prime}-j\right)(K+G)\left(n_{i}^{\left(j^{\prime}\right)}+N_{\mathrm{cp}}+n\right) / N\right]\left[\mathbf{x}_{i}^{\left(j^{\prime}\right)}\right]_{n}$, so $\mathbf{x}_{i, \text { new }}^{\left(j, j^{\prime}\right)}$ actually corresponds to $\mathbf{x}_{i}^{\left(j^{\prime}\right)}$ but with its frequency response circularly shifted down by $\left(j^{\prime}-j\right)(K+G)$ carriers (up to a factor), i.e., $\mathbf{F}_{N} \mathbf{x}_{i, \text { new }}^{\left(j, j^{\prime}\right)}=$ $\exp \left[\iota 2 \pi\left(j^{\prime}-j\right)(K+G)\left(n_{i}^{\left(j^{\prime}\right)}+N_{\mathrm{cp}}\right) / N\right] \mathbf{J}_{\left(j^{\prime}-j\right)(K+G)}$
$\mathbf{F}_{N} \mathbf{x}_{i}^{\left(j^{\prime}\right)}$, where $\mathbf{J}_{m}$ is the $N \times N$ circular shift matrix given by $\mathbf{J}_{m}=\left[\left[\mathbf{I}_{N}\right]_{:, m: N-1},\left[\mathbf{I}_{N}\right]_{:, 0: m-1}\right]$. This last property will be important if one wants to avoid multiband interference. To see this, it is important to realize that if $h_{i}^{(j)}[n ; l]$ and $h_{i, \text { new }}^{\left(j, j^{\prime}\right)}[n ; l]$ have a maximal Doppler spread of $f_{\mathrm{D}}$, then $\tilde{\mathbf{H}}_{i}^{(j)}=\mathbf{F}_{N} \mathbf{H}_{i}^{(j)} \mathbf{F}_{N}^{H}$ and $\tilde{\mathbf{H}}_{i, \text { new }}^{\left(j, j^{\prime}\right)}=\mathbf{F}_{N} \mathbf{H}_{i, \text { new }}^{\left(j, j^{\prime}\right)} \mathbf{F}_{N}^{H}$ are (approximately) circularly banded with some bandwidth $B=\left\lceil f_{\mathrm{D}} N T\right\rceil+1$, a property that can even be enforced by simple time-domain windowing [8]-[11]. In the extreme case of time-invariant channels, $\tilde{\mathbf{H}}_{i}^{(j)}$ and $\tilde{\mathbf{H}}_{i, \text { new }}^{\left(j, j^{\prime}\right)}$ will even be diagonal $(B=1)$, with the frequency responses of $h_{i}^{(j)}[n ; l]$ and $h_{i, \text { new }}^{\left(j, j^{\prime}\right)}[n ; l]$ on the diagonal, respectively. As a result, if we assume that iii) the guard $G /(N T)$ is larger than or equal to the maximal Doppler spread $f_{\mathrm{D}}$, then we know that $G \geq B-1$. As a result, $\mathbf{y}_{i}^{(j)}$ only depends on the $i$-th OFDM symbol of the $j$-th subband if the $K$ carriers of interest are chosen. In other words, the multiband interference can be completely removed by transforming the $N \times 1$ baseband time-domain vector $\mathbf{y}_{i}^{(j)}$ by means of an $N$-point unitary DFT and selecting from it the first $K / 2$ and last $K / 2$ carriers, leading to (6) and (7). This concludes the proof.

\section{APPENDIX C}

In Appendix A, we have derived that

$$
\mathbf{x}_{i}^{(j)}=\mathbf{F}_{N}^{H} \mathbf{T s}_{i}^{(j)}=\mathbf{C P F}_{K}^{H} \mathbf{s}_{i}^{(j)},
$$

where $\mathbf{C}$ and $\mathbf{P}$ are defined in Appendix A. Similarly, we can show that

$$
\mathbf{z}_{i}^{(j)}=\mathbf{T}^{T} \mathbf{F}_{N} \mathbf{y}_{i}^{(j)}=\mathbf{F}_{K} \mathbf{P}^{T} \mathbf{C} \mathbf{y}_{i}^{(j)} .
$$

Joining these two expressions with (7), we obtain

$$
\begin{aligned}
\mathbf{z}_{i}^{(j)} & =\mathbf{T}^{T} \mathbf{F}_{N} \mathbf{H}_{i}^{(j)} \mathbf{F}_{N}^{H} \mathbf{T s}_{i}^{(j)}+\boldsymbol{\eta}_{i}^{(j)} \\
& =\mathbf{F}_{K} \mathbf{P}^{T} \mathbf{C H}_{i}^{(j)} \mathbf{C} \mathbf{P} \mathbf{F}_{K}^{H} \mathbf{s}_{i}^{(j)}+\boldsymbol{\eta}_{i}^{(j)} \\
& =\mathbf{F}_{K} \boldsymbol{\Lambda}_{i}^{(j)} \mathbf{F}_{K}^{H} \mathbf{s}_{i}^{(j)}+\boldsymbol{\eta}_{i}^{(j)} .
\end{aligned}
$$

From $\Lambda_{i}^{(j)}=\mathbf{P}^{T} \mathbf{C H}_{i}^{(j)} \mathbf{C P}$, it is then easy to see that $\boldsymbol{\Lambda}_{i}^{(j)}$ is the $K \times K$ quasi-circular channel matrix given by $\left[\boldsymbol{\Lambda}_{i}^{(j)}\right]_{k, k^{\prime}}=\lambda_{i}^{(j)}\left[k ;\left(k-k^{\prime}\right) \bmod K\right]$ with $\lambda_{i}^{(j)}[k ; l]$ the $N / K$ times downsampled version of $c[n] \circledast h_{i}^{(j)}[n ; l] \circledast c[n]$. This concludes the proof.

\section{APPENDIX D}

Let us define $\mathbf{D}_{q}$ as the $K \times K$ circular matrix whose first column is the frequency response of the $q$-th BEM function, $\mathbf{D}_{q}=\mathbf{F}_{K} \operatorname{diag}\left(\mathbf{b}_{q}\right) \mathbf{F}_{K}^{H}$, and $\boldsymbol{\Delta}_{q}$ as the $K \times K$ diagonal matrix whose diagonal is the frequency response of the BEM coefficient vector related to the $q$-th BEM function, $\boldsymbol{\Delta}_{q}=\operatorname{diag}\left(\mathbf{F}_{\lambda} \mathbf{c}_{i, q}^{(j)}\right)$, with $\mathbf{F}_{\lambda}=\left[\sqrt{K} \mathbf{F}_{K}\right]_{:, 0: L_{\lambda}-1}$ and $\mathbf{c}_{i, q}^{(j)}=\left[c_{i, q}^{(j)}[0], \ldots, c_{i, q}^{(j)}\left[L_{\lambda}-1\right]\right]^{T}$. We can then express $\check{\mathbf{z}}_{i, m}^{(j)}$ as a function of $\check{\mathbf{s}}=\left[\check{\mathbf{s}}_{0}^{T}, \ldots, \check{\mathbf{s}}_{M-1}^{T}\right]^{T}$ as

$$
\check{\mathbf{z}}_{i, m}^{(j)}=\sum_{q=0}^{Q-1} \check{\mathbf{D}}_{q, m} \check{\boldsymbol{\Delta}}_{q} \check{\mathbf{s}}+\check{\mathbf{d}}_{i, m}^{(j)},
$$


where $\check{\mathbf{d}}_{i, m}^{(j)}$ includes all the interference terms and depends on the unknown symbols, the channel, and the noise. Further, $\check{\mathbf{D}}_{q, m}$ is the $\left(K_{\mathrm{t}} / M-2 D\right) \times K_{\mathrm{t}}$ matrix given by $\left[\tilde{\mathbf{D}}_{q, m}\right]_{:, m K_{\mathrm{t}} / M:(m+1) K_{\mathrm{t}} / M-1=}^{=}$ $\left[\mathbf{D}_{q}\right]_{k_{m}+D: k_{m}+K_{\mathrm{t}} / M-D-1, k_{m}: k_{m}+K_{\mathrm{t}} / M-1}, \quad$ and $\check{\boldsymbol{\Delta}}_{q}$ is the $K_{\mathrm{t}} \times K_{\mathrm{t}}$ diagonal matrix given by

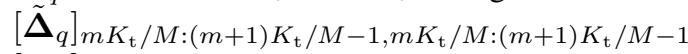
$\left[\boldsymbol{\Delta}_{q}\right]_{k_{m}: k_{m}+K_{\mathrm{t}} / M-1, k_{m}: k_{m}+K_{\mathrm{t}} / M-1}$.

Rewriting (26) as a function of $\mathbf{c}_{i}^{(j)}=$ $\left[\mathbf{c}_{i, 0}^{(j) T}, \ldots, \mathbf{c}_{i, Q-1}^{(j) T}\right]^{T}$, we obtain

$$
\check{\mathbf{z}}_{i, m}^{(j)}=\check{\mathbf{D}}_{m} \check{\mathbf{S}}_{i}^{(j)}+\check{\mathbf{d}}_{i, m}^{(j)},
$$

where $\check{\mathbf{D}}_{m}=\left[\check{\mathbf{D}}_{0, m}, \ldots, \check{\mathbf{D}}_{Q-1, m}\right]$ and $\check{\mathbf{S}}=\mathbf{I}_{Q} \otimes$ $\left[\operatorname{diag}(\check{\mathbf{s}}) \mathbf{F}_{\lambda}\right]$. Stacking the results for all clusters, $\check{\mathbf{z}}_{i}^{(j)}=$ $\left[\check{\mathbf{z}}_{i, 0}^{(j) T}, \ldots, \check{\mathbf{z}}_{i, M-1}^{(j) T}\right]^{T}$, we finally arrive at (10), where $\check{\mathbf{d}}_{i}^{(j)}$ is similarly defined as $\check{\mathbf{z}}_{i}^{(j)}$, and where $\check{\mathbf{D}}=\left[\check{\mathbf{D}}_{0}^{T}, \ldots, \check{\mathbf{D}}_{M-1}^{T}\right]^{T}$. This concludes the proof.

\section{REFERENCES}

[1] J. H. Park Jr., "LPI techniques in the underwater acoustic channel," in IEEE Military Communications Conference, Monterey, Canada, October 1986, pp. 10.5.1-10.5.5.

[2] M. Stojanovic, J. G. Proakis, J. A. Rice, and M. D. Green, "Spread spectrum underwater acoustic telemetry," in IEEE Oceans conference 1998, Nice, France, 1998, pp. 650-654.

[3] S. Azou, G. Burel, L. Le Duff, and C. Pistre, "Sea trial results of a chaotic direct-sequence spread spectrum underwater communication system," in IEEE Oceans conference 2003, San Diego, California, USA, September 2003, pp. 1539-1546.

[4] M. Heinemann, A. Larraza, and K. B. Smith, "Experimental studies of applications of time-reversal acoustics to noncoherent underwater communications," J. Acoustical Society of America, vol. 113, no. 6, pp. 3111-3116, June 2003.

[5] H. S. Dol, F. P. A. Benders, and B. Quesson, "Covert underwater communication with marine mammal sounds," in UDT Europe 2008, Glasgow, United Kingdom, June 2008.

[6] T. C. Yang and W.-B. Yang, "Performance analysis of direct-sequence spread-spectrum underwater acoustic communications with low signalto-noise-ratio input signals," J. Acoustical Society of America, vol. 123 , no. 2, pp. 842-855, February 2008.

[7] E. Sangfelt, B. Nilsson, and J. Israelsson, "Covert underwater communication experiments using DSSS and turbo equalization," in UDT Europe 2008, Glasgow, United Kingdom, June 2008.

[8] W. G. Jeon, K. H. Chang, and Y. S. Cho, "An equalization technique for orthogonal frequency-division multiplexing systems in time-variant multipath channels," IEEE Trans. Commun., vol. 47, no. 1, pp. 27-32, January 1999

[9] P. Schniter, "Low-complexity equalization of OFDM in doubly-selective channels," IEEE Trans. Signal Processing, vol. 52, no. 4, pp. 1002-1011, April 2004.

[10] L. Rugini, P. Banelli, and G. Leus, "Simple equalization of time-varying channels for OFDM," IEEE Commun. Lett., vol. 9, no. 7, pp. 619-621, July 2005.

[11] — "Low-complexity banded equalizers for OFDM systems in Doppler spread channels," EURASIP Journal on Applied Signal Processing, vol. 2006, pp. Article ID 67 404, 13 pages, 2006.

[12] S. Coatelan and A. Glavieux, "Design and test of a multicarrier transmission system on the shallow water acoustic channel," in OCEANS 1994, Brest, France, September 1994, pp. III/472-III/477.

[13] M. Stojanovic, "Low complexity OFDM detector for underwater acoustic channels," in OCEANS 2006, Boston, Massachusetts, USA, September 2006.

[14] B. Li, S. Zhou, M. Stojanovic, and L. Freitag, "Pilot-tone based ZPOFDM demodulation for an underwater acoustic channel," in OCEANS 2006, Boston, Massachusetts, USA, September 2006.

[15] B. Li, S. Zhou, M. Stojanovic, L. Freitag, and P. Willet, "Non-uniform Doppler compensation for zero-padded OFDM over fast-varying underwater acoustic channels," in OCEANS 2007, Vancouver, Canada, 2007.
[16] B. Li, S. Zhou, J. Huang, and P. Willet, "Scalable OFDM design for underwater acoustic communications," in ICASSP 2008, Las Vegas, Nevada, USA, 2008.

[17] M. K. Tsatsanis and G. B. Giannakis, "Modeling and equalization of rapidly fading channels," International Journal of Adaptive Control and Signal Processing, vol. 10, no. 2/3, pp. 159-176, March 1996.

[18] Z. Tang, R. C. Cannizzaro, G. Leus, and P. Banelli, "Pilot-assisted timevarying channel estimation for OFDM systems," IEEE Trans. Signal Processing, vol. 55, no. 5, pp. 2226-2238, May 2007.

[19] R. Kolic, "Ultra wideband - the next-generation wireless connection,”Technology@Intel Magazine, February/March 2004, available at www.intel.com/technology/magazine/communications/wi02042.pdf.

[20] C. Heegard and S. B. Wicker, Turbo Coding. Springer, 1998.

[21] M. Tüchler, A. C. Singer, and R. Koetter, "Minimum mean squared error equalization using a priori information," IEEE Trans. Signal Processing, vol. 50, no. 3, pp. 673-683, March 2002.

[22] R. Otnes and M. Tüchler, "Iterative channel estimation for turbo equalization of time-varying frequency-selective channels," IEEE Trans. Wireless Commun., vol. 3, no. 6, pp. 1918-1923, November 2004.

[23] K. Fang and G. Leus, "Low-complexity block turbo equalization for OFDM systems in time-varying channels," in Proc. IEEE International Conference on Acoustics, Speech, and Signal Processing (ICASSP), Honolulu, Hawaii, April 2007, pp. III/445-III/448.

[24] K. Fang, L. Rugini, and G. Leus, "Iterative channel estimation and turbo equalization for time-varying ofdm systems," in Proc.IEEE International Conference on Acoustics, Speech, and Signal Processing (ICASSP 2008), Las Vegas, Nevada, USA, March/April 2008, to appear.

[25] A. P. Kannu and P. Schniter, "MSE-optimal training for linear timevarying channels," in Proc. IEEE International Conference on Acoustics, Speech, and Signal Processing (ICASSP), Philadelphia, Pennsylvania, March 2005, pp. III/789-III/792.

[26] X. Ma, G. B. Giannakis, and S. Ohno, "Optimal training for block transmissions over doubly-selective fading channels," IEEE Trans. Signal Processing, vol. 51, no. 5, pp. 1351-1366, May 2003.

[27] P. A. van Walree, T. Jenserud, and M. Smedsrud, "A discrete-time channel simulator driven by measured scattering functions," IEEE J. Select. Areas Commun., 2008, accepted.

[28] P. van Walree, E. Sangfelt, and G. Leus, "Multicarrier spread spectrum for covert acoustic communications," in OCEANS 2008, Quebec City, Canada, September 2008.

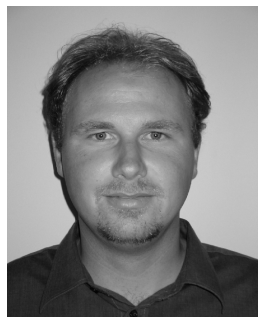

Geert Leus was born in Leuven, Belgium, in 1973. He received the electrical engineering degree and the $\mathrm{PhD}$ degree in applied sciences from the Katholieke Universiteit Leuven, Belgium, in June 1996 and May 2000, respectively. He has been a Research Assistant and a Postdoctoral Fellow of the Fund for Scientific Research - Flanders, Belgium, from October 1996 till September 2003. During that period, Geert Leus was affiliated with the Electrical Engineering Department of the Katholieke Universiteit Leuven, Belgium. Currently, Geert Leus is an Associate Professor at the Faculty of Electrical Engineering, Mathematics and Computer Science of the Delft University of Technology, The Netherlands. During the summer of 1998, he visited Stanford University, and from March 2001 till May 2002 he was a Visiting Researcher and Lecturer at the University of Minnesota. His research interests are in the area of signal processing for communications. Geert Leus received a 2002 IEEE Signal Processing Society Young Author Best Paper Award and a 2005 IEEE Signal Processing Society Best Paper Award. He is the Vice-Chair of the IEEE Signal Processing for Communications Technical Committee, and an Associate Editor for the IEEE Transactions on Signal Processing and the EURASIP Journal on Applied Signal Processing. In the past, he has served on the Editorial Board of the IEEE Signal Processing Letters and the IEEE Transactions on Wireless Communications.

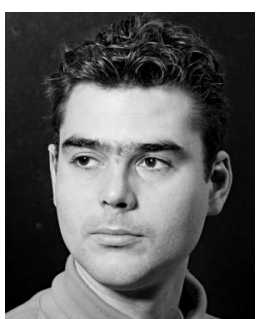

Paul van Walree received the M.Sc. and Ph.D. degrees in (solid-state) physics from Utrecht University, the Netherlands, in 1992 and 1997, respectively. Since 1998 he has been with the Netherlands Organisation for Applied Scientific Research TNO, where he currently holds the position of senior scientist.

His research interests include digital underwater communications, channel characterization, and acoustic signal processing. 